\title{
Stability of Relativistic Force-Free Jets
}

\section{Citation}

Narayan, Ramesh, Jason Li, and Alexander Tchekhovskoy. 2009. “Stability of

Relativistic Force-Free Jets." The Astrophysical Journal 697 (2) (May 14): 1681-1694. doi:10.1088/0004-637x/697/2/1681.

\section{Published Version}

doi:10.1088/0004-637X/697/2/1681

\section{Permanent link}

http://nrs.harvard.edu/urn-3:HUL.InstRepos:26526402

\section{Terms of Use}

This article was downloaded from Harvard University's DASH repository, and is made available under the terms and conditions applicable to Other Posted Material, as set forth at http:// nrs.harvard.edu/urn-3:HUL.InstRepos:dash.current.terms-of-use\#LAA

\section{Share Your Story}

The Harvard community has made this article openly available.

Please share how this access benefits you. Submit a story.

Accessibility 


\title{
STABILITY OF RELATIVISTIC FORCE-FREE JETS
}

\author{
RAMESh NARAYAN ${ }^{1}, \mathrm{JASON} \mathrm{Li}^{2}$, AND ALEXANDER TCHEKHOVSKOY ${ }^{1}$ \\ ${ }^{1}$ Harvard-Smithsonian Center for Astrophysics, 60 Garden Street, Cambridge, MA 02138, USA; rnarayan@cfa.harvard.edu \\ ${ }^{2}$ Department of Astrophysical Sciences, Princeton University, Peyton Hall, Ivy Lane, Princeton, NJ 08544, USA \\ Received 2009 January 28; accepted 2009 March 24; published 2009 May 13
}

\begin{abstract}
We consider a two-parameter family of cylindrical force-free equilibria, modeled to match numerical simulations of relativistic force-free jets. We study the linear stability of these equilibria, assuming a rigid impenetrable wall at the outer cylindrical radius $R_{j}$. Equilibria in which the Lorentz factor $\gamma(R)$ increases monotonically with increasing radius $R$ are found to be stable. On the other hand, equilibria in which $\gamma(R)$ reaches a maximum value at an intermediate radius and then declines to a smaller value $\gamma_{j}$ at $R_{j}$ are unstable. A feature of these unstable equilibria is that poloidal field line curvature plays a prominent role in maintaining transverse force balance. The most rapidly growing mode is an $m=1$ kink instability which has a growth rate $\sim\left(0.4 / \gamma_{j}\right)\left(c / R_{j}\right)$. The $e$-folding length of the equivalent convected instability is $\sim 2.5 \gamma_{j} R_{j}$. For a typical jet with an opening angle $\theta_{j} \sim$ few $/ \gamma_{j}$, the mode amplitude grows only weakly with increasing distance from the base of the jet. The growth is much slower than one might expect from a naive application of the Kruskal-Shafranov stability criterion.
\end{abstract}

Key words: galaxies: jets - instabilities - MHD

\section{INTRODUCTION}

Relativistic jets in astrophysical sources have been known for many decades. Although their enormous power, large Lorentz factor, and strong collimation have been widely studied, these phenomena still lack an accepted explanation. An even greater mystery is the remarkable coherence and apparent stability of jets over very large length scales. This is the topic of the present paper.

The most promising models of relativistic jets involve acceleration and collimation by magnetic fields with footpoints attached to a spinning black hole/neutron star or an accretion disk. The forced rotation of the field lines induces a strong toroidal component of the magnetic field, which is responsible for accelerating the jet (e.g., Narayan et al. 2007; Tchekhovskoy et al. 2008, hereafter TMN08; and references therein). In this picture the toroidal component of the field dominates over other field components.

According to the well known Kruskal-Shafranov (KS) criterion (e.g., Bateman 1978), cylindrical magnetohydrodynamic (MHD) configurations in which the toroidal field dominates are violently unstable to the $m=1$ kink instability (also called the screw instability). The KS criterion for instability is

$$
\left|\frac{B_{\phi}}{B_{p}}\right|>\frac{2 \pi R_{j}}{z},
$$

where $B_{\phi}$ and $B_{p}$ are the toroidal and poloidal magnetic field strengths, $R_{j}$ is the cylindrical radius, and $z$ the length of the system. Typical jet models, including those described in this paper (see Section 2.2), have $B_{\phi} \sim \gamma_{j} B_{p}$, where $\gamma_{j} \gg 1$ is the Lorentz factor of the jet, and they have jet angles $\theta_{j} \sim R_{j} / z \sim$ few $/ \gamma_{j}$. Substituting these scalings in Equation (1), it is obvious that the KS instability criterion is easily satisfied in relativistic jets. We therefore expect astrophysical jets to be violently unstable, as argued for example by Begelman (1998) and Li (2000). Yet, jets in nature are apparently quite stable. How is this possible?

Many authors have investigated this question. They have used jet models with a variety of velocity profiles, geometrical shapes, composition, and boundary conditions (Kadomtsev 1966; Bateman 1978; Ferrari et al. 1978; Benford 1981; Payne \& Cohn 1985), and applied both analytical and numerical methods (Istomin \& Pariev 1996; Begelman 1998; Lyubarskii 1999; Li 2000; Lery et al. 2000; Appl et al. 2000; Tomimatsu et al. 2001; Mizuno et al. 2007; Moll et al. 2008; McKinney \& Blandford 2009). As a result of this large body of work, several kinds of unstable modes have been identified: reflection modes, Kelvin-Helmholtz modes, current-carrying modes, etc. Unfortunately, it is difficult to synthesize the results to extract universal principles.

A fruitful approach in this field is to reduce relativistic jet models to their barest minimum. One such approach is to consider force-free jet models in which one ignores the inertia and pressure of the plasma and considers only charges, currents, and fields. The force-free approximation is valid whenever the energy density in fields dominates over matter energy density, as in pulsar magnetospheres (Goldreich \& Julian 1969; Ruderman \& Sutherland 1975). The force-free approximation is valid also in relativistic MHD jets, at least inside the fast magnetosonic surface (e.g., Tchekhovskoy et al. 2009; Lyubarsky 2009).

Theoretical studies of force-free jets have led to the identification of two distinct stability criteria. In a detailed analysis, Istomin \& Pariev (1996) showed that cylindrical force-free jets in which $B_{z}$ is independent of $R$ are stable. Lyubarskii (1999) considered models with nonconstant $B_{z}$ and showed that forcefree jets are unstable if

$$
\frac{d B_{z}}{d R}<0,
$$

i.e., if the poloidal field decreases with increasing distance from the axis. We refer to Equation (2) as the IPL criterion for instability. On the other hand, Tomimatsu et al. (2001; TMT) showed via an approximate analysis ${ }^{3}$ that force-free jets are unstable if

$$
\left|\frac{B_{\phi}}{B_{p}}\right|>\frac{|\Omega| R}{c},
$$

\footnotetext{
3 They effectively restricted their analysis to the region of the jet inside the light cylinder. Therefore, the flow speeds they considered were at best only quasi-relativistic.
} 
where $\Omega$ is the angular velocity of the field line. This criterionthe TMT criterion-differs from the KS criterion (1) in that it explicitly accounts for rotation. It is also apparently very different from the IPL criterion.

We describe, in this paper, a class of force-free cylindrical jet equilibria that closely match the numerical force-free jet simulations reported in TMN08, including the important effect of poloidal field curvature. Within the context of force-free jets from rigidly rotating stars, we believe that this two-parameter family of equilibria is generic and fairly complete. We study the stability properties of these equilibria and attempt to relate our results to the KS, IPL, and TMT criteria (Equations (1)-(3)).

In Section 2, we summarize the numerical simulation results of TMN08 (Section 2.1) and we describe an analytical forcefree jet model that matches the simulation data very closely (Section 2.2). In Section 3, we carry out a linear stability analysis of these equilibria and show that the linear modes of the system are obtained by solving an eigenvalue problem involving two coupled differential equations, with appropriate boundary conditions. In Section 4, we numerically solve the equations and identify the unstable modes in the system. We also derive an approximate estimate for the growth rate of the instability. We conclude in Section 5 with a summary and discussion.

We use $(r, \theta, \phi)$ for spherical coordinates and $(R, \phi, z)$ for cylindrical coordinates. The idealized jet equilibria we consider are axisymmetric and cylindrical, with rigid wall boundary conditions at radius $R=R_{j}$. Each equilibrium is defined in terms of two parameters, $R_{m}$ and $\gamma_{m}$, as described in Section 2.2. When $R_{m} / R_{j}<1$, the Lorentz factor $\gamma$ reaches a maximum value equal to $\gamma_{m}$ at $R=R_{m}$ and decreases to a smaller value $\gamma_{j}$ at $R=R_{j}$. On the other hand, when $R_{m} / R_{j}>1, \gamma$ increases monotonically out to $R=R_{j}$. These two types of models are described in Sections 2.2.1 and 2.2.2, and Figures 1 and 2, and their stability properties are studied in Section 4.

\section{FORCE-FREE JET EQUILIBRIUM}

\subsection{Structure of Force-Free Jets}

TMN08 considered a rigidly rotating star of unit radius $(r=1)$ surrounded by a differentially rotating infinitely thin disk extending from $R=1$ outward. The star was threaded by a uniform radial magnetic field $B_{r}$, and the disk was threaded by a power-law distribution of vertical field,

$$
B_{z} \propto R^{\nu-2} \text {. }
$$

Using a relativistic force-free code (Gammie et al. 2003; McKinney \& Gammie 2004; McKinney 2006; Mignone \& McKinney 2007; Tchekhovskoy et al. 2007), TMN08 numerically evolved the system and obtained the equilibrium configuration of the magnetic field.

Following TMN08, we will call the field lines that emerge from the star as the "jet" and the field lines from the disk as the "wind." The critical field line that emerges from the star-disk boundary defines the boundary between the jet and the wind. This boundary starts off at $\theta=\pi / 2$ at the surface of the star $(r=R=1)$ but decreases to smaller values of $\theta$ with increasing $r$ (or $z)$.

We are primarily interested in the "jet" - the bundle of field lines attached to the central star. Since all of these field lines rotate at the angular velocity $\Omega$ of the star, the Alfvén surface for these lines takes the form of a cylinder-the "light cylinder"with radius

$$
R_{A}=c / \Omega
$$

Field lines become strongly toroidal once they are outside the Alfvén surface, which is where most of the collimation and acceleration occurs (TMN08).

TMN08 showed that the structure of the jet is strongly affected by the radial pressure profile of the region surrounding the jet. Specifically, if we write the radial variation of the confining pressure as $p \propto r^{-\alpha}$, the jet properties are determined by the value of $\alpha$. In the numerical experiments, the pressure was caused by a force-free disk wind, and $\alpha$ was determined by the index $v$ (Equation (4)) according to

$$
\alpha=2(2-v)
$$

At distance $z$ along the axis, the cylindrical radius $R_{j}$ of the jet is approximately given by

$$
R_{j} \sim z^{\alpha / 4}
$$

For all $\alpha<4$, the jet collimates as it moves away from the star (Lynden-Bell 2006). As a result, at a sufficiently large distance from the central star $(r \gg 1)$, the jet is nearly cylindrical in shape.

In the asymptotic nearly cylindrical region of the jet, force balance in the $R$ direction is described by the following equation (TMN08; see Equation (23) below for the cylindrical version of this equation):

$$
\frac{d}{d R}\left(\frac{B^{2}-E^{2}}{8 \pi}\right)+\left(\frac{B_{\phi}^{2}-E^{2}}{4 \pi R}\right)+\left(\frac{B_{p}^{2}-E^{2}}{4 \pi R_{c}}\right)=0,
$$

where $B$ is the total magnetic field strength, $B_{\phi}$ is the toroidal field strength, and $B_{p}$ is the poloidal field strength:

$$
B=\sqrt{B_{p}^{2}+B_{\phi}^{2}}, \quad B_{p}=\sqrt{B_{R}^{2}+B_{z}^{2}} .
$$

The electric field $\vec{E}$ is given by

$$
\vec{E}=-(\Omega R / c) \hat{\phi} \times \vec{B},
$$

where $\Omega$ is the angular velocity of the field line. The electric field has only a poloidal component: $E_{p}=\Omega R B_{p} / c$.

Each of the three terms on the left-hand side of Equation (8) represents a force in the $-R$ direction. The quantity $\left(B^{2}-\right.$ $\left.E^{2}\right) / 8 \pi$ is the pressure of the force-free fluid in the comoving frame; therefore, the first term describes the inward force due to the gradient of pressure. The second term arises from the toroidal curvature of the field line. The toroidal magnetic field $B_{\phi}$ contributes an inward force due to "hoop stress," while the poloidal electric field $E$ contributes an outward force. ${ }^{4}$ The third term in (8) gives analogous contributions from the poloidal curvature of the field line, where $R_{c}$ is the poloidal radius of curvature; once again there is an inward force due to the poloidal magnetic hoop stress and an outward force due to the electric field. Note that the contributions involving $E$ are important only for relativistic flows. In standard nonrelativistic MHD, one neglects these terms and keeps only the terms involving $B$.

TMN08 showed that models with $\alpha>2$, i.e., $v<1$, are good analogs of relativistic jets found in nature, especially gammaray burst jets. The main features of the numerical jet solutions

\footnotetext{
4 As Equation (10) shows, $\vec{E}$ is directly proportional to $\Omega$, so the latter term results from rotation and may loosely be viewed as a sort of "centrifugal force" (V. Beskin 2007, private communication).
} 
are as follows (see Figure 1 below). (1) $B_{p}$ is essentially constant inside the jet, showing hardly any variation with $R$. As we show in Appendix A, this is required for force-free jet solutions that smoothly connect to the central compact object. ${ }^{5}$ (2) Since $\Omega$ is constant and $E \propto \Omega R B_{p}$ (Equation (10)), we have $E^{2} \propto R^{2}$. (3) $B_{\phi}$ is almost equal to $E$ and so $B_{\phi}$ also varies primarily as $R^{2}$. (4) $\left|B_{\phi}\right|$ is slightly larger than $E$ with $B_{\phi}^{2}-E^{2} \propto R^{4}$. Because of this property, the first two terms in Equation (8) both represent inward forces. Therefore, (5) the third term in Equation (8), which involves the poloidal curvature of the field line, is important for force balance. This is the only outward force in the balance equation-it is outward because $E$ is of order $B_{\phi}$ and is much greater than $B_{p}$ outside the light cylinder. This force has to balance the other two terms.

The velocity of a force-free flow is usually identified with the drift velocity,

$$
\frac{\vec{v}}{c}=\frac{\vec{E} \times \vec{B}}{B^{2}}
$$

and the Lorentz factor is defined correspondingly. Since $\vec{E} \cdot \vec{B}=$ 0 , it is easily shown that

$$
\gamma^{2}=\frac{B^{2}}{B^{2}-E^{2}}
$$

TMN08 derived two approximate relations for $\gamma$,

$$
\begin{gathered}
\gamma_{1}=\left[1+(\Omega R / c)^{2}\right]^{1 / 2}, \\
\gamma_{2}=\left(3 R_{c} / R\right)^{1 / 2},
\end{gathered}
$$

and they showed that the net $\gamma$ of the fluid is given to good accuracy by the following simple formula (this result is derived later; see Equation (29)):

$$
\frac{1}{\gamma^{2}}=\frac{1}{\gamma_{1}^{2}}+\frac{1}{\gamma_{2}^{2}}
$$

Along each field line, $\gamma$ is initially determined mainly by rotation, and so $\gamma \approx \gamma_{1}$. This is a region of efficient acceleration which TMN08 called the first acceleration regime. However, beyond a certain distance from the star, the effect of poloidal field line curvature becomes important, and $\gamma$ switches to the less efficient $\gamma_{2}$, the second acceleration regime.

Relatively near the star, all field lines in the jet are in the first acceleration regime and $\gamma(R)$ behaves like $\gamma_{1}$ (Equation (13)). Specifically, $\gamma$ increases more or less linearly with $R$ and reaches its maximum value at the edge of the jet at $R=R_{j}$. However, when we consider the jet at a larger distance from the star, some of the field lines have already switched to the second acceleration regime, where $\gamma \sim \gamma_{2} \propto 1 / R$ (see Equation (14), coupled with Equation (22) below). In this case, the maximum Lorentz factor occurs at some radius $R_{m}$ inside the jet, not at the boundary; we have $\gamma \sim \gamma_{1} \propto R$ for $R \lesssim R_{m}$ and $\gamma \sim \gamma_{2} \propto R^{-1}$ for $R_{m} \lesssim R<R_{j}$. TMN08 discuss in detail the physics of the two acceleration regimes.

\footnotetext{
5 Asymptotic force-free jet configurations with nonconstant profiles of $B_{p}$ are certainly possible (Lyubarskii 1999), but there exists no solution that would smoothly connect them to the compact object.
}

\subsection{Analytical Jet Model}

The axisymmetric numerical jet models described in Section 2.1 have magnetic and electric field components that are functions of both $R$ and $z$. This is not convenient for linear perturbation analysis. Since the numerical models are nearly cylindrical at a large distance (i.e., $d R / d z \ll 1$ ), we now consider an idealized jet equilibrium model which is perfectly cylindrical and in which all quantities are functions only of $R$. We choose the following specific functional forms:

$$
\begin{gathered}
B_{0 R}=0 \\
B_{0 \phi}=-\left[2\left(\gamma_{m}^{2}-1\right)\left(R / R_{m}\right)^{2}+\left(R / R_{m}\right)^{4}\right]^{1 / 2} \equiv-f(R) \\
B_{0 z}=\exp \left[-3 R^{2} / 4\left(\gamma_{m}^{2}-1\right) R_{m}^{2}\right] \equiv g(R) \\
E_{0 R}=-\left[2\left(\gamma_{m}^{2}-1\right)\right]^{1 / 2}\left(R / R_{m}\right) \equiv-h(R) \\
E_{0 \phi}=0 \\
E_{0 z}=0 \\
R_{c}=2\left(\gamma_{m}^{2}-1\right) R_{m}^{2} / 3 R
\end{gathered}
$$

The zeros in the subscripts are meant to indicate that all these quantities refer to the unperturbed model. The model has two parameters, $\gamma_{m}$ and $R_{m}$, whose meanings are explained below. For simplicity, we have chosen units such that $B_{0 z}=1$ at the jet axis $(R=0)$. Also, we have assumed that $B_{0 z}$ and $\Omega$ are positive, so both $B_{0 \phi}$ and $E_{0 R}$ are negative, i.e., magnetic field lines are swept backward with respect to the rotation and the electric field is pointed radially inward. With this choice of signs, the three functions $f(R), g(R)$, and $h(R)$ are positive. Note that, in all cases of interest, $g(R)$ is practically equal to unity. The particular exponential form given in Equation (18) is designed to handle small higher-order terms in the force balance Equation (23), but the deviations of $g(R)$ from unity are tiny and unimportant. ${ }^{6}$

By direct substitution it is easily verified that the above model satisfies the radial force balance Equation (8). Under cylindrical symmetry, this equation takes the form

$\frac{d}{d R}\left(\frac{B_{0 \phi}^{2}+B_{0 z}^{2}-E_{0 R}^{2}}{8 \pi}\right)+\left(\frac{B_{0 \phi}^{2}-E_{0 R}^{2}}{4 \pi R}\right)+\left(\frac{B_{0 z}^{2}-E_{0 R}^{2}}{4 \pi R_{c}}\right)=0$.

The first two terms are positive, i.e., both represent inward forces, with the first term providing twice as much force as the second. The third term is negative and its magnitude is equal to the sum of the other two terms.

An important feature of the above model is that the outward force from the third term involves the poloidal curvature radius $R_{c}$. Technically, a perfectly cylindrical model has $R_{c} \rightarrow \infty$. To get around this problem, we treat $R_{c}$ as an externally imposed

\footnotetext{
6 As a test, in the stability analysis described later we have done the calculations both with the full expression for $g(R)$ given in Equation (18) and with the simpler choice $g(R)=1$. The results are practically the same.
} 
property of the solution which is adjusted so as to reproduce the poloidal curvature force present in the numerical jet model. In other words, even though we have straightened out field lines in the $z$-direction by enforcing cylindrical geometry, we still retain the effect of poloidal curvature by means of an artificial external force. This procedure is analogous to the widely used shearing sheet approximation in accretion disk studies (Goldreich \& Tremaine 1978; Narayan et al. 1987) in which fluid streamlines are straightened out in the azimuthal direction, but the effect of azimuthal curvature is still retained via a Coriolis force. Note that, apart from the extra term due to poloidal field curvature, Equation (8) is identical to the standard balance condition derived in other papers in the literature, e.g., Equation (6) in Istomin \& Pariev (1996) or Equation (16) in Lyubarskii (1999).

To get a better idea of the nature of the above analytical model, we now make a couple of simplifications. As already mentioned, $B_{0 z}$ is practically independent of $R$ inside the jet. Also, for highly relativistic jets, we invariably have $B_{\phi}^{2}-E^{2} \ll B_{\phi}^{2}$. We therefore replace Equations (17), (18), and (19) by the following simpler formulae:

$$
\begin{gathered}
B_{0 z} \approx 1, \\
B_{0 \phi} \approx E_{0 R}=-\left[2\left(\gamma_{m}^{2}-1\right)\right]^{1 / 2}\left(R / R_{m}\right), \\
B_{0 \phi}^{2}-E^{2}=\left(R / R_{m}\right)^{4} .
\end{gathered}
$$

By Equation (10), the angular velocity of rotation of the field lines is given by

$$
\Omega=-c E_{0 R} / B_{0 z} R \approx \sqrt{2\left(\gamma_{m}^{2}-1\right)}\left(c / R_{m}\right),
$$

and is the same for all field lines, as required for a rigidly rotating star at the base of the jet. ${ }^{7}$ Using these simpler expressions, we obtain the following result for the Lorentz factor:

$$
\begin{aligned}
\frac{1}{\gamma^{2}(R)}= & \frac{B_{0}^{2}-E_{0}^{2}}{B_{0}^{2}}=\frac{B_{0 \phi}^{2}+B_{0 z}^{2}-E_{0 R}^{2}}{B_{0 \phi}^{2}+B_{0 z}^{2}} \\
& \approx \frac{1+\left(R / R_{m}\right)^{4}}{1+2\left(\gamma_{m}^{2}-1\right)\left(R / R_{m}\right)^{2}} \\
& \approx \frac{1}{1+(\Omega R / c)^{2}}+\frac{R}{3 R_{c}},
\end{aligned}
$$

where we have made use of Equations (22) and (27). We thus reproduce the result given earlier in Equation (15).

The simple analytical model described here can reproduce all the features seen in numerical simulations of force-free jets. Figure 1 shows numerical results obtained by TMN08 for their "fiducial" model ( $\nu=0.75$ or $\alpha=2.5$ ). Panels (a) and (b) correspond to a relatively near region of the jet at $z=10^{2}$, where the jet is entirely in the first acceleration regime. Panels (c) and (d) correspond to a more distant region at $z=10^{7}$, where part of the jet has made the transition to the second acceleration regime (the region where $\gamma$ decreases with increasing $R$ ). In each panel, the abscissa gives the cylindrical radius $R$ normalized by

\footnotetext{
7 Since we have designed our analytical model to match the numerical models of TMN08, all of our models have constant $\Omega(R)$. It would be straightforward to generalize the model to nonconstant $\Omega(R)$, using additional parameters.
}
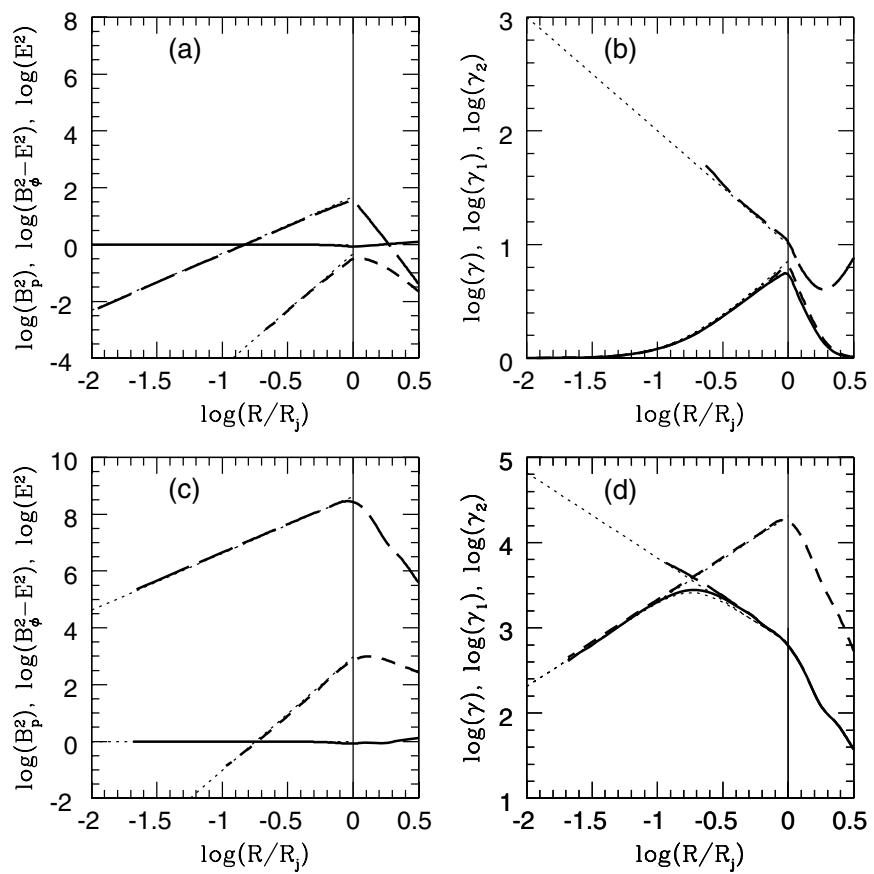

Figure 1. Numerical results from a force-free jet simulation with $\alpha=2.5$ ("fiducial model" corresponding to $v=0.75$ in TMN08). Panel (a): field components as functions of normalized cylindrical radius $R / R_{j}$ at $z=10^{2}$. The solid horizontal line shows $B_{p}^{2}(R) / B_{p}^{2}(0)$, the short-dashed line shows $\left[B_{\phi}^{2}(R)-E^{2}(R)\right] / B_{p}^{2}(0)$ and the long-dashed line shows $E^{2}(R) / B_{p}^{2}(0)$. The dotted lines are the corresponding results for the analytical model with $\gamma_{m}=6$, $R_{m}=1.2$ (Model A, Section 2.2.1). The vertical solid line shows the boundary between the jet and the external confining medium. Panel (b): Lorentz factor $\gamma(R)$ (solid line), and the two approximations, $\gamma_{1}(R)$ (short-dashed line) and $\gamma_{2}(R)$ (long-dashed line), at the same $z$. The dotted lines are from the analytical model. Panels (c) and (d): similar to (a) and (b), but at $z=10^{7}$. The dotted lines in these panels correspond to the analytical model with $\gamma_{m}=2600, R_{m}=0.18$ (Model C, Section 2.2.2).

the local "jet radius" $R_{j}$, which is the cylindrical radius of the field line that separates the jet from the surrounding disk wind.

In the analytical model, it is easily shown that $\gamma$ reaches a maximum at $R=R_{m}$ and that its value at this radius is equal to $\gamma_{m}$. Thus, the two model parameters $R_{m}$ and $\gamma_{m}$ allow us to control the basic features of the equilibrium. To obtain a jet that is entirely in the first acceleration regime (similar to Figures 1(a) and (b)), we must choose $R_{m}>R_{j}$, while for a jet that is partly in the second acceleration regime (Figures 1(c) and (d)), we require $R_{m}<R_{j}$. We consider these two cases in the following subsections.

\subsection{1. $R_{m}>R_{j}$ : Maximum Lorentz Factor Located at the Jet Boundary}

A jet in which all field lines are in the first acceleration regime has its maximum Lorentz factor at the boundary of the jet, $R=R_{j}$. This corresponds to choosing $R_{m}>R_{j}$ in the analytical model, so that the term $\left(R / R_{m}\right)^{4}$ in the numerator of Equation (28) can be neglected. In this case, the profile of $\gamma$ has two segments: the region of the jet inside the light cylinder $\left(R<R_{A}=c / \Omega\right)$ which is not accelerated very much, and the region outside the light cylinder which has $\gamma$ increasing linearly with radius,

$$
\gamma(R) \approx \begin{cases}1, & R<R_{A}=R_{m} / \sqrt{2\left(\gamma_{m}^{2}-1\right)}, \\ R / R_{A} \approx\left(R / R_{j}\right) \gamma_{j}, & R_{A}<R<R_{j},\end{cases}
$$




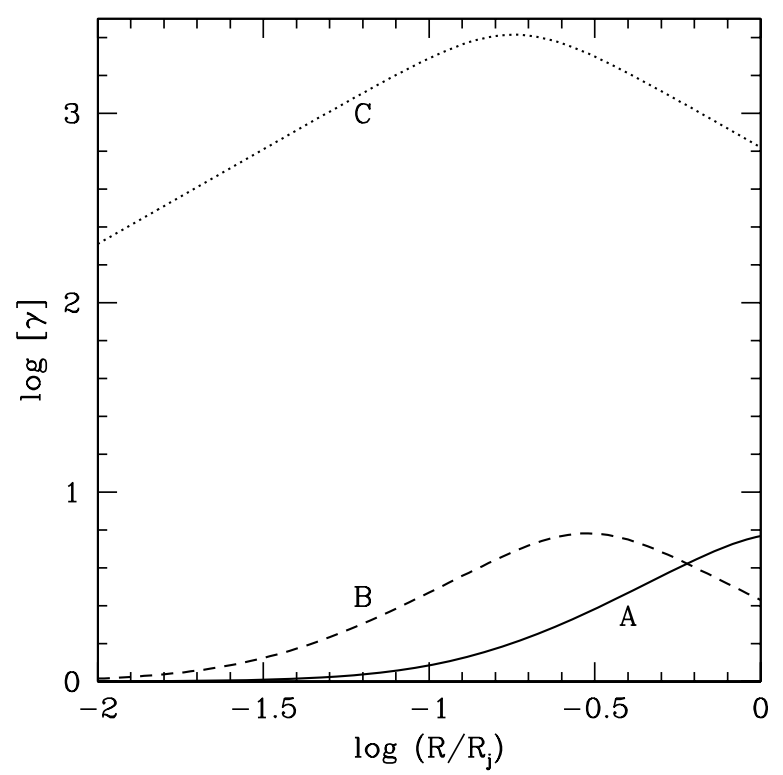

Figure 2. Profiles of $\gamma$ vs. $R / R_{j}$ for the analytic Models $\mathrm{A}, \mathrm{B}$, and C.

where $\gamma_{j}$ is the Lorentz factor at the jet boundary,

$$
\gamma_{j} \approx \gamma_{1}\left(R_{j}\right)=\left[1+2\left(\gamma_{m}^{2}-1\right)\left(R_{j} / R_{m}\right)^{2}\right]^{1 / 2} .
$$

The dotted lines in panels (a) and (b) in Figure 1 show the dependences of various quantities as a function of $R / R_{j}$ for a model with $\gamma_{m}=6$ and $R_{m}=1.2 R_{j}$. The agreement with the numerical simulation results at $z=10^{2}$ is striking. We call the analytical model with this particular choice of $\gamma_{m}$ and $R_{m}$ Model A:

$$
\text { Model A : } \quad \gamma_{m}=6, \quad R_{m}=1.2 R_{j} .
$$

The solid line in Figure 2 shows the variation of $\gamma$ as a function of $R$ for this model.

\subsection{2. $R_{m}<R_{j}$ : Maximum Lorentz Factor Located Inside the Jet}

As we described in Section 2.1, a jet in which some field lines have switched to the second acceleration regime has its maximum Lorentz factor inside the jet. This means $R_{m}<R_{j}$. In this case, the Lorentz factor $\gamma_{j}$ at the jet boundary is roughly equal to

$$
\gamma_{j} \approx \gamma_{2}\left(R_{j}\right)=\left[2\left(\gamma_{m}^{2}-1\right)\right]^{1 / 2} R_{m} / R_{j}
$$

Now the profile of $\gamma$ has three segments:

$$
\gamma(R) \approx \begin{cases}1, & R<R_{A}, \\ R / R_{A} \approx\left(R / R_{m}\right) \gamma_{m}, & R_{A}<R<R_{m}, \\ \left(R_{m} / R\right) \gamma_{m} \approx\left(R_{j} / R\right) \gamma_{j}, & R_{m}<R<R_{j} .\end{cases}
$$

The dotted lines in panels (c) and (d) of Figure 1 show model results corresponding to $\gamma_{m}=2600$ and $R_{m}=0.18 R_{j}$. We find excellent agreement with the numerical results at $z=10^{7}$. We call the analytical model with these values of $\gamma_{m}$ and $R_{m}$ Model C. For completeness we also consider a less extreme model called Model B in which $\gamma_{m}=6$ and $R_{m}=0.3 R_{j}$ :

$$
\begin{array}{lll}
\text { Model B: } & \gamma_{m}=6, & R_{m}=0.3 R_{j}, \\
\text { Model C: } & \gamma_{m}=2600, & R_{m}=0.18 R_{j} .
\end{array}
$$

The dashed and dotted lines in Figure 2 show the variations of $\gamma$ as a function of $R$ for these two models.

\section{LINEAR PERTURBATION ANALYSIS}

We now consider linear perturbations of the cylindrical equilibrium described in Section 2.2. The unperturbed state has magnetic and electric fields

$$
\begin{aligned}
& \vec{B}_{0}=B_{0 R} \hat{R}+B_{0 \phi} \hat{\phi}+B_{0 z} \hat{z}, \\
& \vec{E}_{0}=E_{0 R} \hat{R}+E_{0 \phi} \hat{\phi}+E_{0 z} \hat{z},
\end{aligned}
$$

where the various components are given by the expressions in Equations (16)-(21). As mentioned previously, we choose $B_{0 z}$ and $\Omega$ to be positive, so $B_{0 \phi}$ and $E_{0 R}$ are negative. The unperturbed current and electric charge are

$$
\vec{J}_{0}=\frac{c}{4 \pi} \vec{\nabla} \times \vec{B}_{0}=\frac{c}{4 \pi}\left[-\left(\frac{d B_{0 z}}{d R}+\frac{B_{0 z}}{R_{c}}\right) \hat{\phi}+\frac{1}{R} \frac{d}{d R}\left(R B_{0 \phi}\right) \hat{z}\right],
$$

$$
\rho_{0}=\frac{1}{4 \pi} \vec{\nabla} \cdot \vec{E}_{0}=\frac{1}{4 \pi R} \frac{d}{d R}\left(R E_{0 R}\right)+\frac{1}{4 \pi} \frac{E_{0 R}}{R_{c}} .
$$

Note that we have included terms involving $R_{c}$ in the unperturbed current and charge density. These terms describe the contributions of poloidal field curvature to the quantities $\vec{\nabla} \times \vec{B}_{0}$ and $\vec{\nabla} \cdot \vec{E}_{0}$, respectively. By including these terms, we retain the forces associated with poloidal field curvature without actually having curved field lines in the model.

\subsection{The Eigenvalue Problem}

We now consider small perturbations. Let us write the perturbed electric field as $\vec{E}=\vec{E}_{0}+\vec{E}_{1}$, where $\vec{E}_{1}$ is a small perturbation of the form

$\vec{E}_{1}=\left[E_{1 R}(R) \hat{R}+E_{1 \phi}(R) \hat{\phi}+E_{1 z}(R) \hat{z}\right] \exp (-i \omega t+i m \phi+i k z)$.

Let us similarly write $\vec{B}=\vec{B}_{0}+\vec{B}_{1}, \vec{J}=\vec{J}_{0}+\vec{J}_{1}, \rho=\rho_{0}+\rho_{1}$. Each of these small perturbations can be expressed in terms of the perturbed electric field via Maxwell's equations. From

$$
\frac{1}{c} \frac{\partial \vec{B}}{\partial t}=-\vec{\nabla} \times \vec{E}
$$

we obtain

$$
\vec{B}_{1}=-\frac{i c}{\omega} \vec{\nabla} \times \vec{E}_{1}
$$

From

$$
\frac{1}{c} \frac{\partial \vec{E}}{\partial t}=\vec{\nabla} \times \vec{B}-\frac{4 \pi}{c} \vec{J}
$$

we obtain

$$
\vec{J}_{1}=\frac{i \omega}{4 \pi} \vec{E}_{1}-\frac{i c^{2}}{4 \pi \omega} \vec{\nabla} \times\left(\vec{\nabla} \times \vec{E}_{1}\right)
$$

Finally, from

$$
\vec{\nabla} \cdot \vec{E}=4 \pi \rho
$$

we obtain

$$
\rho_{1}=\frac{1}{4 \pi} \vec{\nabla} \cdot \vec{E}_{1}
$$

Since the perturbed system is force-free, it must satisfy $\vec{E} \cdot \vec{B}=0$. The zeroth-order terms satisfy this trivially (as they 
should). The first-order terms give the condition $\vec{E}_{1} \cdot \vec{B}_{0}+\vec{E}_{0} \cdot \vec{B}_{1}$ $=0$. Substituting for the various quantities, this condition allows us to solve for $E_{1 \phi}$ in terms of $E_{1 z}$ :

$$
E_{1 \phi}=C_{1} E_{1 z},
$$

where the function $C_{1}$ is given by

$$
C_{1}=\frac{\omega R g-c m h}{\omega R f-c k R h} .
$$

Successive differentiations give

$$
\begin{gathered}
E_{1 \phi}^{\prime}=C_{1} E_{1 z}^{\prime}+C_{1}^{\prime} E_{1 z}, \\
E_{1 \phi}^{\prime \prime}=C_{1} E_{1 z}^{\prime \prime}+2 C_{1}^{\prime} E_{1 z}^{\prime}+C_{1}^{\prime \prime} E_{1 z},
\end{gathered}
$$

where primes refer to derivatives with respect to $R$

We now consider the force balance condition: $\rho \vec{E}+(1 / c) \vec{J} \times \vec{B}$ $=0$. The zeroth-order terms give

$$
\rho_{0} \vec{E}_{0}+\frac{1}{c} \vec{J}_{0} \times \vec{B}_{0}=0,
$$

which is simply the equilibrium force balance condition (23). Notice that the poloidal curvature terms in $\vec{J}_{0}$ and $\rho_{0}$ are necessary to satisfy equilibrium in the unperturbed solution. From the first-order terms in the force balance equation we obtain

$$
\rho_{1} \vec{E}_{0}+\rho_{0} \vec{E}_{1}+\frac{1}{c} \vec{J}_{1} \times \vec{B}_{0}+\frac{1}{c} \vec{J}_{0} \times \vec{B}_{1}=0 .
$$

The $\hat{\phi}$ component of this equation gives a relation between $E_{1 \phi}^{\prime}$, $E_{1 \phi}, E_{1 z}^{\prime}$, and $E_{1 z}$. Eliminating $E_{1 \phi}^{\prime}$ using Equation (50), we obtain a first-order differential equation for $E_{1 z}(R)$ :

$$
D_{1} E_{1 z}^{\prime}+D_{2} E_{1 z}+D_{3} E_{1 R}=0
$$

where $D_{1}, D_{2}$, and $D_{3}$ are functions of $R$. The expressions are relatively long and we give them in Appendix B. The $\hat{z}$ component of Equation (53) has no new information; it just gives back Equation (48). The $\hat{R}$ component, however, gives a new relation between the various components of $\vec{E}_{1}$ and their derivatives. Eliminating $E_{1 \phi}^{\prime}, E_{1 \phi}^{\prime \prime}, E_{1 z}^{\prime}$, and $E_{1 z}^{\prime \prime}$ using Equations (50), (51), (54), and the derivative of (54), we obtain a differential equation for $E_{1 R}(R)$ :

$$
D_{4} E_{1 R}^{\prime}+D_{5} E_{1 z}+D_{6} E_{1 R}=0,
$$

where $D_{4}, D_{5}$, and $D_{6}$ are again functions of $R$ and are given in Appendix B.

We have thus reduced the linear mode analysis problem to a pair of first-order differential equations, Equations (54) and (55), for $E_{1 z}(R)$ and $E_{1 R}(R)$. For convenience, we write down the two equations again:

$$
\begin{aligned}
& E_{1 z}^{\prime}=-\frac{D_{2}}{D_{1}} E_{1 z}-\frac{D_{3}}{D_{1}} E_{1 R}, \\
& E_{1 R}^{\prime}=-\frac{D_{5}}{D_{4}} E_{1 z}-\frac{D_{6}}{D_{4}} E_{1 R} .
\end{aligned}
$$

These equations constitute an eigenvalue problem, where $\omega$ is the eigenvalue. By numerically solving the equations with appropriate boundary conditions, we obtain $\omega$ for given values of $m$ and $k$.

The singular points of the equations are located at the radii where $D_{1}(R)$ and $D_{4}(R)$ vanish. Anticipating later discussion, we write down here the expression for the quantity $D_{1} D_{4}$ :

$$
\begin{aligned}
D_{1} D_{4}= & -\frac{g}{\omega R} \\
& \times\left[\frac{(\omega R f-c k R h)^{2}+(\omega R g-m c h)^{2}-(c k R g-m c f)^{2}}{(\omega R f-c k R h)}\right] .
\end{aligned}
$$

Also, from Equation (11), the perturbed velocity is

$$
\frac{\vec{v}_{1}}{c}=\frac{\vec{E}_{1} \times \vec{B}_{0}+\vec{E}_{0} \times \vec{B}_{1}}{B^{2}} .
$$

In component form this gives

$$
\begin{gathered}
\frac{v_{1 R}}{c}=\frac{g E_{1 \phi}+f E_{1 z}}{f^{2}+g^{2}}, \\
\frac{v_{1 \phi}}{c}=-\frac{g E_{1 R}-h B_{1 z}}{f^{2}+g^{2}}, \\
\frac{v_{1 z}}{c}=-\frac{f E_{1 R}+h B_{1 \phi}}{f^{2}+g^{2}} .
\end{gathered}
$$

We now consider boundary conditions. A physically valid perturbation will be well behaved on the axis $(R=0)$ and will satisfy suitable boundary conditions at the jet boundary $(R=$ $\left.R_{j}\right)$. The condition on the axis is different for axisymmetric $(m=0)$ and nonaxisymmetric $(|m| \geqslant 1)$ perturbations, so we consider each of these cases in turn. At the jet boundary, we assume that the jet is constrained by a "rigid wall" and we write down the corresponding boundary condition. In the following, we employ the specific forms of $f(R), g(R)$, and $h(R)$ given in Equations (17), (18), and (19).

\subsection{Boundary Condition on the Axis: $m=0$}

Setting $m=0$ and substituting the expressions for $f(R)$, $g(R)$, and $h(R)$ in $D_{1}-D_{6}$, we find that the leading terms of the differential equations, Equations (56) and (57), at small $R$ are given by

$$
\begin{gathered}
E_{1 z}^{\prime}=\frac{a_{z z}}{R} E_{1 z}+a_{z R} E_{1 R}, \\
E_{1 R}^{\prime}=\frac{a_{R z}}{R^{2}} E_{1 z}+\frac{a_{R R}}{R} E_{1 R},
\end{gathered}
$$

where

$$
\begin{gathered}
a_{z z}=-\frac{2 \omega}{c k}, \\
a_{z R}=\frac{i\left(c^{2} k^{2}-\omega^{2}\right)}{c^{2} k}, \\
a_{R z}=\frac{4 i \omega}{k(c k-\omega)}, \\
a_{R R}=\frac{(c k+2 \omega)}{c k} .
\end{gathered}
$$


Requiring the perturbation to be analytic as $R \rightarrow 0$ immediately gives the following solution near the axis:

$$
\begin{gathered}
E_{1 z}=K R^{2}, \\
E_{1 R}=-K \frac{2 i c}{(c k-\omega)} R,
\end{gathered}
$$

where $K$ is an arbitrary normalization constant.

\subsection{Boundary Condition on the Axis: $|m|>0$}

When $m \neq 0$, we obtain

$$
\begin{gathered}
a_{z z}=0, \\
a_{z R}=\frac{i m A(c k-\omega)}{\left(A c m-\omega R_{m}\right)}, \\
a_{R z}=-\frac{i m\left(A c m-\omega R_{m}\right)}{A(c k-\omega)}, \\
a_{R R}=-1,
\end{gathered}
$$

where the constant $A$ is defined to be

$$
A=\left[2\left(\gamma_{m}^{2}-1\right)\right]^{1 / 2} .
$$

The physically relevant solution close to the axis is then

$$
\begin{gathered}
E_{1 z}=K R^{|m|}, \\
E_{1 R}=-K \operatorname{sgn}(m) \frac{i\left(A c m-\omega R_{m}\right)}{A(c k-\omega)} R^{|m|-1} .
\end{gathered}
$$

\subsection{Boundary Condition at the Jet Boundary: Rigid Wall}

We assume that our cylindrical jet is terminated at $R=R_{j}$ by a rigid impenetrable wall. By impenetrable we mean that no energy flows across this boundary, i.e., the Poynting flux lies in the $\phi-z$ plane. Equivalently, the velocity vector has no radial component.

The equilibrium Poynting flux of course lies in the $\phi-z$ plane. The perturbed Poynting flux is proportional to $\vec{E}_{1} \times \vec{B}_{0}+\vec{E}_{0} \times \vec{B}_{1}$. Since $\vec{E}_{0}$ is parallel to $\hat{R}$, the term $\vec{E}_{0} \times \vec{B}_{1}$ is automatically in the $\phi-z$ plane. The term $\vec{E}_{1} \times \vec{B}_{0}$ will also be in this plane if $\vec{E}_{1}$ is precisely radial, i.e., both $E_{1 \phi}$ and $E_{1 z}$ vanish. By Equation (48), $E_{1 \phi}$ is proportional to $E_{1 z}$. We thus obtain the following boundary condition at the outer wall:

$$
E_{1 z}=0, \quad R=R_{j} .
$$

\section{NUMERICAL RESULTS}

We have computed frequencies of modes by numerically solving the differential equations, Equations (56) and (57), along with the boundary conditions described in Sections 3.2-3.4. For each choice of $k$ and $m$, a countable infinity of solutions exists which may be ordered by the number of zeros of $\operatorname{Re}\left[E_{1 z}(R)\right]$, not counting zeros at the boundaries. ${ }^{8}$ The lowest-order solution (the "fundamental mode") is such that $\operatorname{Re}\left[E_{1 z}(R)\right]$ has no zeros between $R=0$ and $R=R_{j}$, the next solution has one zero

\footnotetext{
$8 \operatorname{Re}()$ stands for the real part of a complex quantity.
}

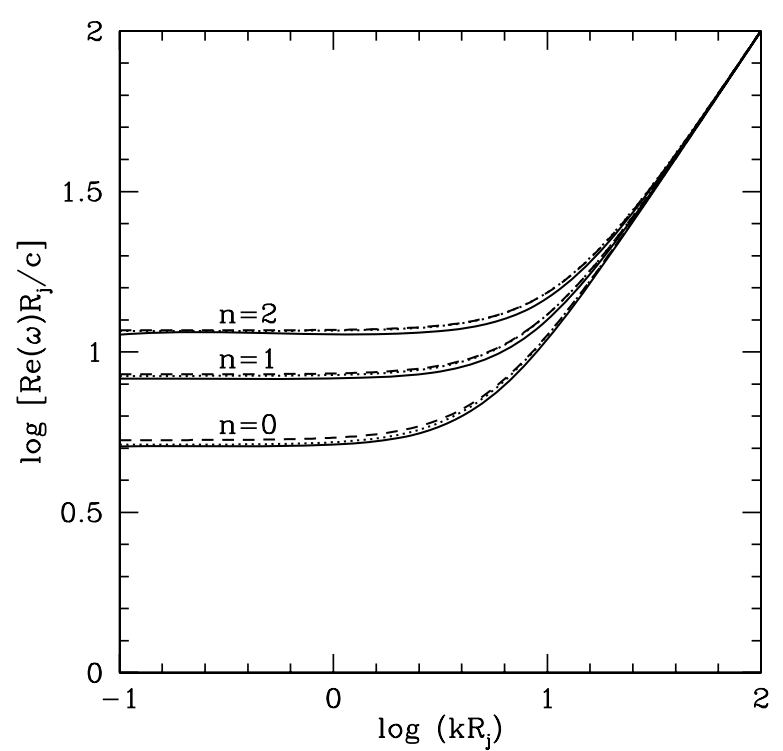

Figure 3. Dispersion relation for axisymmetric modes $(m=0)$ in Models A (solid lines), B (dashed lines), and C (dotted lines). The curves corresponding to radial mode numbers $n=0,1$, and 2 are labeled. All the modes are stable.

inside the jet, and so on. In the following we identify each mode by its radial mode number $n$ which we define to be the number of zeros. As one might expect, the mode frequency increases with increasing $n$.

We solve for the frequencies via a shooting method. We start with a guess value of $\omega$, make use of the expressions given in Section 3.2 or Section 3.3 (depending on the value of $m$ ) to set up the initial solution at small $R$, and integrate Equations (56) and (57) to $R=R_{j}$. We then adjust $\omega$ in the complex plane until the outer boundary condition given in Section 3.4 is satisfied. The only subtle point is that the quantities $D_{1}$ and $D_{4}$ appear in the denominators of various coefficients in Equations (56) and (57), and so their zeros correspond to singularities in the solution. To avoid these singularities, we treat $R$ as a complex variable and integrate the equations over a "safe" trajectory in complex- $R$ space. Since the solution is analytic, the exact track that we follow is unimportant so long as it lies above all singularities in the $R$-plane. Istomin \& Pariev (1996) give a detailed discussion of this point in connection with currentdriven instabilities in force-free jets. The reader is also referred to standard discussions of the topic in plasma physics texts in the context of Landau damping, or Goldreich et al. (1986) for a discussion in the context of accretion disk instabilities.

\subsection{Axisymmetric Modes: $m=0$}

Figure 3 shows the dispersion relation-the variation of $\omega$ with $k$-of axisymmetric modes $(m=0)$. Results are shown for Models A, B, and C (Equations (32), (35), and (36)) for three radial mode numbers: $n=0,1$, and 2 . For all $k$, we find that the mode frequency is real, which means that all these modes are stable.

For large values of $k R_{j}$, the mode frequency asymptotes to $\omega= \pm c k$, so the modes behave like electromagnetic waves moving parallel or antiparallel to the $z$-axis. At small $k$, however, the frequency asymptotes to a constant value. There is thus a minimum frequency for propagating modes inside the jet. The minimum frequency is of order the inverse of the lightcrossing time across a radial wavelength of the mode (e.g., $\omega_{\min } \sim 2 \pi c / R_{j}$ for the mode with $n=0$ ). 


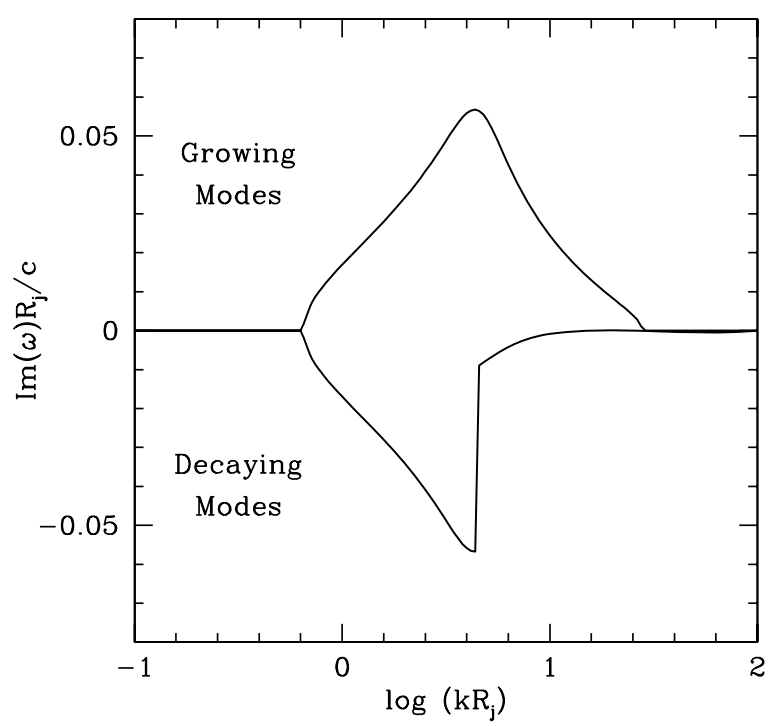

Figure 4. Imaginary part of $\omega$ for modes in Model B with $m=1$ and $n=0$ Growing modes have $\operatorname{Im}(\omega)>0$, while decaying modes have $\operatorname{Im}(\omega)<0$.

We find that the dispersion relations of modes with positive and negative $k$ are not quite the same. The difference arises because the background has a nonzero velocity in the $z$-direction, which breaks the symmetry between waves propagating toward $+z$ and $-z$. The effect is, however, quite weak.

\subsection{Nonaxisymmetric Modes: $m= \pm 1$}

The most interesting modes are those with $m= \pm 1$. These modes are stable in Model A, but unstable in Models B and C.

Figure 4 shows $\operatorname{Im}(\omega)^{9}$ as a function of $k R_{j}$ for a sequence of modes in Model B; the modes correspond to $m=1$ and $n=0$. In this sequence, modes with $k R_{j}<0.65$ and those with $k R_{j}>28$ are stable and have $\omega$ real. However, for $0.65<k R_{j}<28$, we find a pair of modes with complex values of $\omega$. The branch with $\operatorname{Im}(\omega)>0$ corresponds to growing modes, and the branch with $\operatorname{Im}(\omega)<0$ to decaying modes. ${ }^{10}$

Figure 5 shows eigenfunctions corresponding to a few of the growing modes. Plotted is $\operatorname{Re}\left(E_{1 z}\right)$ as a function of the scaled radius $R / R_{j}$. The mode corresponding to $k R_{j}=5$ is representative of all modes with $k R_{j} \lesssim 5$. These modes have eigenfunctions with no zero crossings between $R=0$ and $R=R_{j}$. By our definition, the modes correspond to $n=0$. Each of the remaining eigenmodes in Figure 5 has a pronounced dip in $\operatorname{Re}\left(E_{1 z}\right)$ which causes a zero crossing. These dips result from a singularity in the equations, as we discuss below. If we discount the singularity-induced zero crossings, then these eigenfunctions may also be identified as $n=0$ modes.

Figures 6 and 7 show similar results for Model C. Growing modes (and their decaying counterparts) are present for $m=1$ and all $k R_{j}<2.1 \times 10^{4}$. Modes with $m=-1$ are also unstable (see Figure 6). ${ }^{11}$ Figure 7 shows a few eigenfunctions. All modes with $k R_{j} \lesssim 1.3 \times 10^{3}$ have eigenfunctions with the standard $n=0$ shape (see the mode with $k R_{j}=1.25 \times 10^{3}$ ). For larger values of $k$, the eigenfunctions develop negative spikes due to the presence of a singularity. However, we still view them as $n=0$ modes.

\footnotetext{
$9 \operatorname{Im}()$ refers to the imaginary part of a complex quantity.

10 We discuss at the end of Section 4.2 the reason for the sudden jump in the value of $\operatorname{Im}(\omega)$ in the decaying branch.

11 In the case of Model B, modes with $m=-1$ appear to be stable, and only the $m=+1$ modes show an instability.
}

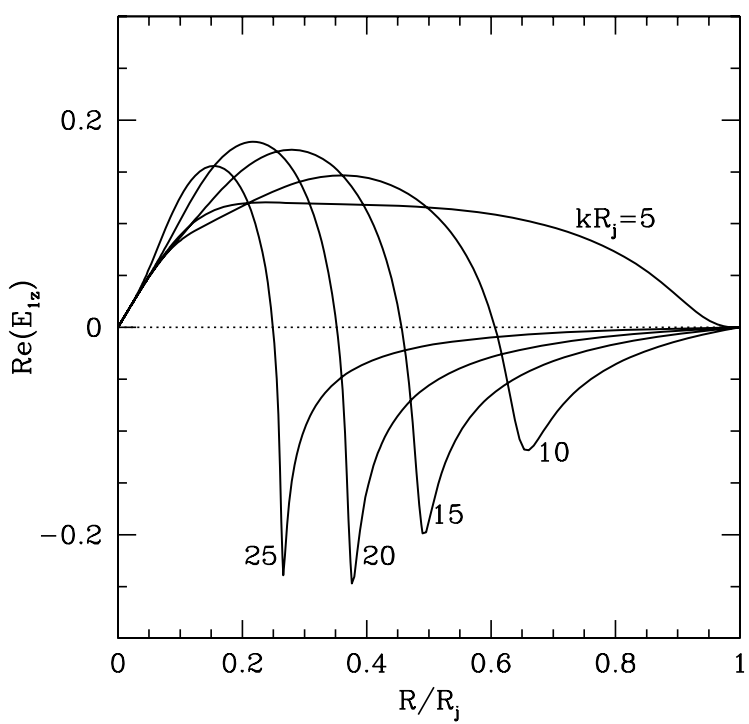

Figure 5. Eigenfunctions corresponding to growing modes in Model B with $m=1, n=0$ and $k R_{j}=5,10,15,20$, and 25. The real part of $E_{1 z}$ is plotted.

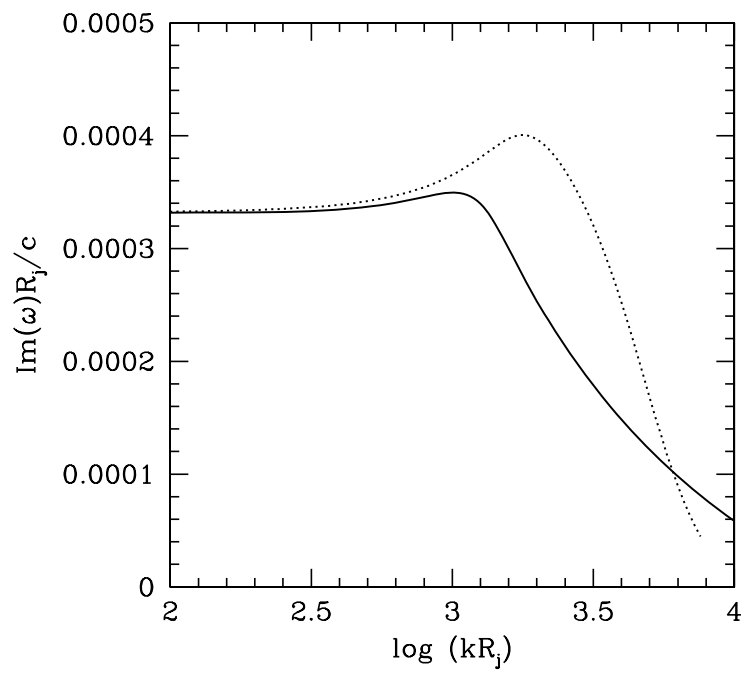

Figure 6. Imaginary part of $\omega$ for growing modes in Model C. The solid line corresponds to modes with $m=1$ and $n=0$ and the dotted line corresponds to modes with $m=-1$ and $n=0$.

We have determined numerically that the singularities which cause the dips in the eigenfunctions are due to zeros in the function $D_{4}(R)$ defined in Section 3.1. This function appears in the denominator of the differential equation, Equation (57), and hence its zeros behave like poles. ${ }^{12}$

Equation (58) gives the analytic form of the quantity $D_{1} D_{4}$. Since the modes of interest to us have $\operatorname{Re}(\omega)$ very nearly equal to $c k$, let us substitute $\omega=c k$ in this equation. Then, setting $D_{1} D_{4}$ equal to zero gives the following relation between the wavenumber $k$ of the mode and the radius $R_{\text {sing }}$ of the singularity:

$$
k R_{\text {sing }}=\frac{m\left[f\left(R_{\text {sing }}\right)+h\left(R_{\text {sing }}\right)\right]}{\left.g\left(R_{\text {sing }}\right)+\operatorname{sgn}(m) \sqrt{g^{2}\left(R_{\text {sing }}\right)+\left[f^{2}\left(R_{\text {sing }}\right)-h^{2}\left(R_{\text {sing }}\right)\right.}\right]} .
$$

Figure 8 shows the position of the singularity $R_{\text {sing }}$ as a function of $k R_{j}$ for modes with $m= \pm 1$ in Models $\mathrm{B}$ and $\mathrm{C}$, as calculated with this equation. For comparison, the dots show

\footnotetext{
12 In contrast, although the function $D_{1}(R)$ appears in the denominator of Equation (56), its zeros correspond to removable singularities in the differential equation.
} 


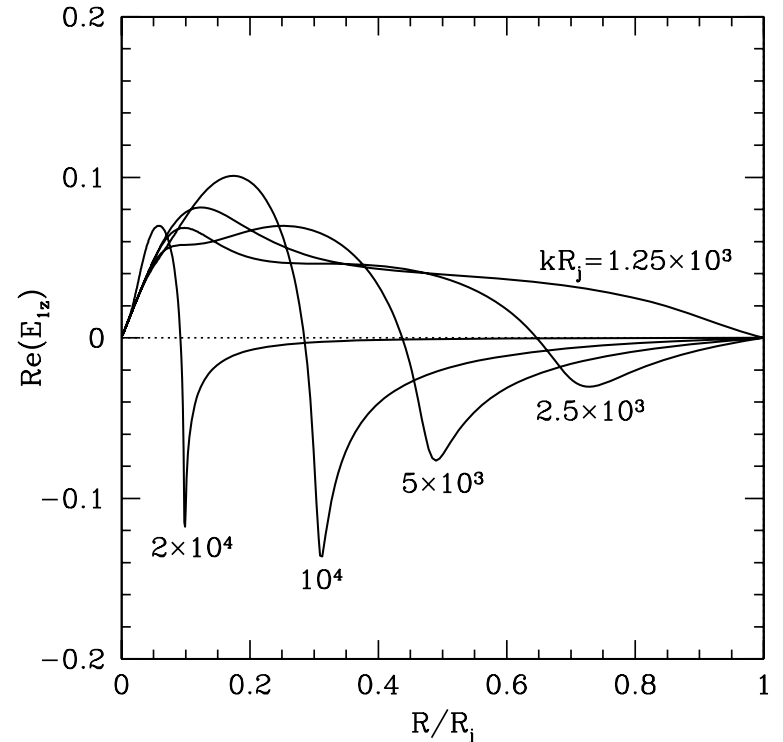

Figure 7. Eigenfunctions corresponding to growing modes in Model $\mathrm{C}$ with $m=1, n=0$, and $k R_{j}=1.25 \times 10^{3}, 2.5 \times 10^{3}, 5 \times 10^{3}, 10^{4}$, and $2 \times 10^{4}$. The real part of $E_{1 z}$ is plotted.

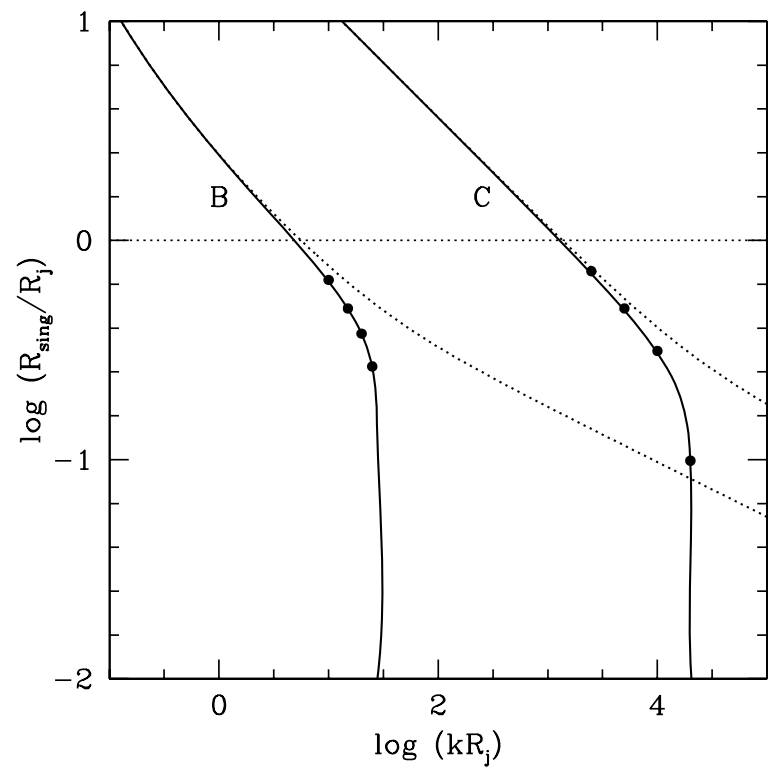

Figure 8. Location of the singularity $R_{\text {sing }}$ as a function of $k R_{j}$ in Models B and $C$, calculated using Equation (79). The solid curves correspond to modes with $m=1$ and $n=0$ and dotted lines to modes with $m=-1$ and $n=0$. The solid dots show the locations of minima in the eigenfunctions plotted in Figures 5 and 7.

the radii at which the functions $\operatorname{Re}\left[E_{1 z}(R)\right]$ reach their minima in the eigenfunctions plotted in Figures 5 and 7 . The agreement between the analytical curve and the dots is excellent, showing that Equation (79) captures the physics of the singularity.

From Figure 8 we see that the singularity lies inside the jet $\left(R_{\text {sing }}<R_{j}\right)$ only for a finite range of $k$ above a certain minimum value. For values of $k$ smaller than this minimum, the singularity is outside the jet (for very small $k$ it is well outside the jet). In the case of Model $B$, the singularity enters the jet from outside when $k R_{j} \sim 5$ and it disappears at $R=0$ when $k R_{j} \sim 28$ (for $m=+1$ ). This is the primary range of $k$ over which an unstable mode is present. At $k R_{j} \sim 28$, the singularity is barely present near the center of the jet and we have a very weakly growing mode. With decreasing $k$, the singularity moves outward and the growth rate of the mode increases (Figure 4). At $k R_{j} \sim 5$, when the singularity reaches the wall, the growth rate is close to its maximum value. At yet smaller values of $k$, the singularity moves outside the outer wall, but its presence is still felt and there is continued instability. The growth rate, however, decreases with decreasing $k$.

A similar pattern is seen in Model C. Unstable modes are present only for $k R_{j} \lesssim 2 \times 10^{4}$. With decreasing $k$ the growth rate increases and reaches its maximum approximately when the singularity reaches the jet boundary $\left(R_{\text {sing }}=R_{j}\right)$, which happens at $k R_{j} \sim 1.3 \times 10^{3}$. In contrast to Model $\mathrm{B}$, however, the growth rate remains large even for smaller values of $k$, and the instability survives as $k \rightarrow 0$.

We finally discuss the peculiar behavior of $\operatorname{Im}(\omega)$ in the decaying branch of modes in Figure 4. As we mentioned earlier, in numerically solving for the eigenvalue we must integrate the differential equations, Equations (56) and (57), along a path in the complex- $R$ plane that lies above the poles in the solution. For growing modes, the pole is located below the real $R$-axis. We can therefore integrate along the real $R$-axis without any difficulty. For decaying modes, however, the pole is above the real $R$-axis and we must choose the integration path with care. If the singularity has $\operatorname{Re}\left(R_{\text {sing }}\right)>R_{j}$, i.e., the singularity is outside the jet, there is no problem and we can simply integrate along the real axis. However, when $0<\operatorname{Re}\left(R_{\text {sing }}\right)<R_{j}$, we have to deform the integration path. In our calculations, we integrated from $R=0$ along a path with $\operatorname{Im}(R)=\operatorname{Re}(R)$ until the point $\operatorname{Im}(R)=\operatorname{Re}(R)=R_{j}$ and we then integrated down to $R=R_{j}$. The jump in $\operatorname{Im}(\omega)$ in Figure 4 is the result of the singularity moving into the jet. To the left of the break, the singularity is located at $R>R_{j}$. Here the eigenvalues of the growing and decaying modes are complex conjugates of each other. However, to the right of the break, the singularity has moved inside the jet $\left(R<R_{j}\right)$ and now the complex conjugate symmetry is broken.

We note that eigenfunctions and eigenvalues of decaying modes are not very meaningful. This can be shown by analyzing the initial value problem along the lines of Landau's treatment of plasma damping. The reader is referred to Istomin \& Pariev (1996) for a detailed discussion of this topic.

\subsection{Why is $m= \pm 1$ Special?}

We have not exhaustively explored modes with $|m|>1$. However, in spot tests with various choices of $m, n$, and $k R_{j}$ in Models A, B, and C, we found all modes to be stable. We believe that, if at all, there are only weakly unstable modes for $|m|>1$; there is no sign of the kind of vigorous instability described in Section 4.2 for modes with $m= \pm 1$. So why is $m= \pm 1$ special? The answer to this question is well known in the magnetic confinement literature (e.g., Bateman 1978). We discuss it briefly here for completeness.

Consider the radial component of the perturbed velocity $v_{1 R}$ near the axis of the jet. Equation (60) gives the expression for $v_{1 R}$ in terms of the perturbed electric field components $E_{1 z}$ and $E_{1 \phi}$, and Equation (48) shows the relation between these two field components. For small values of $R$ near the axis, we have

$$
g(R) \approx 1, \quad f(R) \approx h(R)=A \frac{R}{R_{m}},
$$

where the quantity $A$ is defined in Equation (75), and

$$
C_{1} \approx \frac{\left(\omega R_{m}-A c m\right)}{A R(\omega-c k)} .
$$


Consider first modes with $m=0$. Equation (69) shows that $E_{1 z} \approx K R^{2}$ near the axis. Substituting this in Equation (60) and using the other approximations given above, we find

$$
v_{1 R} \approx \frac{\omega R_{m}}{A(\omega-c k)} K R+\mathcal{O}\left(R^{3}\right) .
$$

By symmetry, the velocity goes to zero on the axis, and the flow consists of a simple radial divergence.

Consider next modes with $m \neq 0$. Using Equation (76) for $E_{1 z}$, we find

$$
v_{1 R} \approx \frac{\left(\omega R_{m}-A c m\right)}{A(\omega-c k)} K R^{|m|-1} \cos m \phi+\mathcal{O}\left(R^{|m|+1}\right),
$$

where we have included $\cos m \phi$ to show the angular dependence of the mode. The leading term goes like $R^{|m|-1}$, which corresponds to $R^{0}$ when $m= \pm 1$. This means that the mode has a finite radial velocity, and hence a finite radial displacement, on the axis when $|m|=1$. The $\cos \phi$ dependence of $v_{1 R}$, coupled with the fact that $v_{1 \phi}$ has the same amplitude but a $\sin \phi$ dependence, ensures that the velocity vector is unique and analytic at $R=0$. By writing the velocity vector in Cartesian coordinates, it is easily seen that the complex phase of $v_{1 R}$ determines the orientation of the velocity vector in the $x-y$ plane. If we consider values of $|m| \geqslant 2$, the velocity vanishes on the axis, just as in the case of $m=0$.

This then reveals what is special about $|m|=1$ modes. These are the only modes in which fluid perturbations communicate across the axis and cause the jet to shift bodily across the axis. In modes with $|m|=1$ the center of mass of the jet itself shifts into a spiral shape, which is the characteristic feature of the kink or screw mode. For all other values of $|m|$, the center of mass remains on the axis and the perturbations are concentrated on the outside.

In helical MHD configurations in the laboratory, the $|m|=1$ kink mode is known to be highly unstable and to be the greatest threat to the stability of equilibria (Bateman 1978). Not surprisingly we see the same feature in our force-free jet equilibria.

\subsection{Growth Rate of the Instability}

The growth rate of the fastest growing mode is a matter of practical interest since it limits the lifetime of an unstable system. As discussed in Section 4.2, for the models we have considered here the most unstable mode generally has a singularity close to the outer wall: $R_{\text {sing }} \sim R_{j}$. Knowing this, we estimate here the fastest growth rate by assuming that the pole is located at $R_{\text {sing }}=1.1 R_{j}$. (We locate the singularity slightly outside the jet, since this speeds up the numerical integrations.)

Given an assumed value of $R_{\text {sing }}$, we can substitute this value in Equation (79) and make use of the expressions for $f(R)$, $g(R)$, and $h(R)$ given in Section 2.2. Recalling that Models B and $C$ are in the regime described in Section 2.2.2, we note that $f^{2}\left(R_{j}\right)-h^{2}\left(R_{j}\right) \gg g^{2}\left(R_{j}\right)$. In addition, $f$ and $h$ are nearly equal to each other and $\gamma_{j}$ is given by Equation (33). We then find that $k R_{j} \approx 1.6 \gamma_{j}$. Also, the real part of the frequency is nearly equal to $c k$. Thus, we estimate

Mode with $R_{\text {sing }}=1.1 R_{\mathrm{j}}: \quad k \approx \frac{1.6 \gamma_{j}}{R_{j}}, \quad \operatorname{Re}(\omega) \approx \frac{1.6 \gamma_{j} c}{R_{j}}$.

These estimates should apply to the fastest growing mode. The mode with the maximum growth rate in Model B has $\operatorname{Re}(\omega) \approx$

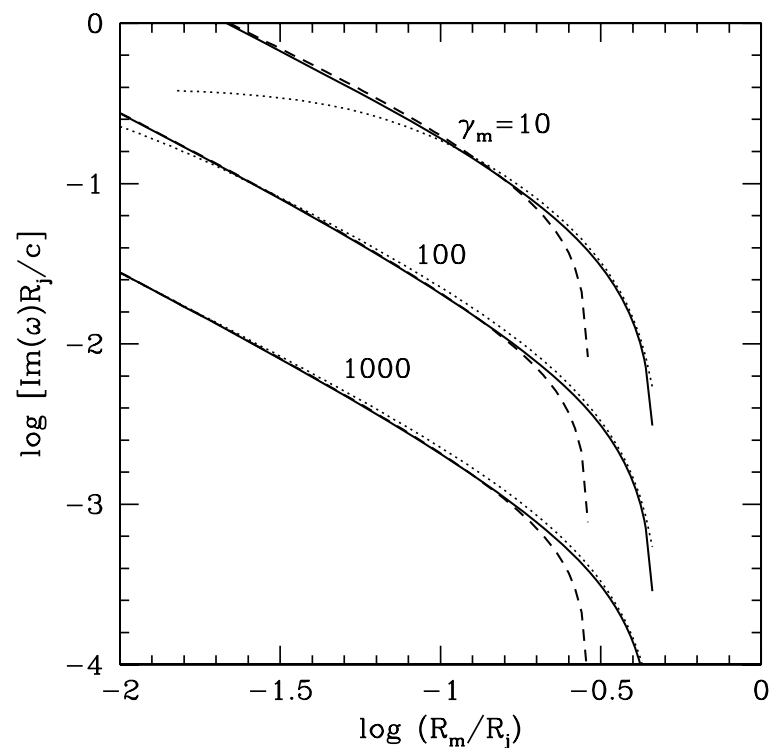

Figure 9. Numerically calculated growth rates of modes with $m=1$ and $R_{\text {sing }}=1.1 R_{j}$. These modes have among the largest growth rates. The solid lines show results for a series of models with fixed $\gamma_{m}$ and varying $R_{m} / R_{j}$. The dotted lines are the growth rates predicted by Equation (85). Note the very good agreement except near the top of the plot, where the models are nonrelativistic. The dashed lines are the numerical growth rates for modes with $k=0$.

$k R_{j}=4.4$. Since Model $\mathrm{B}$ has $\gamma_{j}=2.5$, Equation (84) predicts $k R_{j} \approx 4.0$, which is close. Similarly, the mode with the maximum growth rate in Model $\mathrm{C}$ has $\operatorname{Re}(\omega) \approx k R_{j}=1000$, whereas Equation (85) with $\gamma_{j}=660$ predicts $k R_{j} \approx 1060$. We see that the approximate formula (84) is quite good.

Our numerical results indicate that the growth rate $\operatorname{Im}(\omega)$ of the fastest growing mode is proportional to $\operatorname{Re}(\omega) / \gamma_{j}^{2}$. We also know that unstable modes are present only when $R_{m}<R_{j}$; for instance, Model A with $R_{m}=1.2 R_{j}$ has no unstable modes, whereas Model B with $R_{m}=0.3 R_{j}$ and Model C with $R_{m}=0.18 R_{j}$ both have unstable modes. With these clues in mind, we obtain the following empirical estimate for the growth rate of the fastest growing mode:

$$
\text { Mode with } \mathrm{R}_{\text {sing }}=1.1 \mathrm{R}_{\mathrm{j}}: \quad \operatorname{Im}(\omega) \approx \frac{0.4}{\gamma_{j}}\left(1-\frac{2 R_{m}}{R_{j}}\right) \frac{c}{R_{j}} .
$$

The coefficients 0.4 and 2 are very approximate (to emphasize this, we give their values to only one significant digit). Nevertheless, as we show in Figures 9 and 10, this approximate formula does quite a good job of fitting the numerical results for a wide range of models. The only region of parameter space where the formula fails is when the underlying equilibrium becomes "nonrelativistic" and $\gamma_{j}$ approaches unity. Such models, which are located near the upper end of Figures 9 and 10, have extremely large growth rates.

Although modes with $R_{\text {sing }} \sim R_{j}$ have the largest growth rates, these modes have relatively short wavelengths $\ll R_{j}$ along the $z$-axis (see Equation (84)). With such short wavelengths it is not clear if the instability can grow to a large amplitude. It is therefore interesting to consider modes with $k \rightarrow 0$. Figure 4 shows that Model $\mathrm{B}$ is stable as $k \rightarrow 0$, whereas Figure 6 indicates that the $k=0$ mode in Model $\mathrm{C}$ is nearly as unstable as the fastest growing mode.

The dashed lines in Figures 9 and 10 show numerical results for the growth rates of modes with $k=0$ for various combinations of the model parameters $\gamma_{m}$ and $R_{m}$. For small 


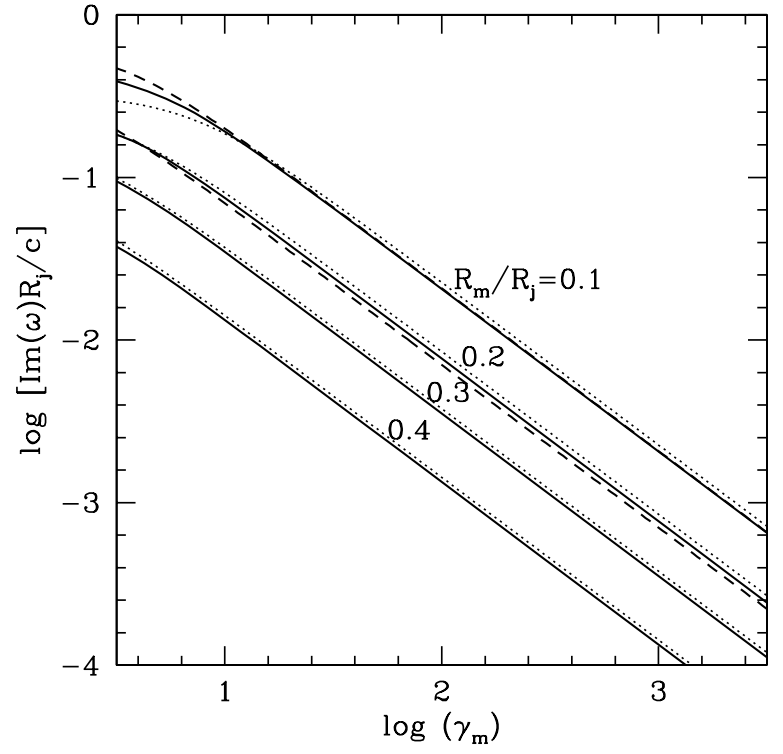

Figure 10. Numerically calculated growth rates of modes with $m=1$ and $R_{\text {sing }}=1.1 R_{j}$. The solid lines show the results for a series of models with fixed $R_{m} / R_{j}$ and varying $\gamma_{m}$. The dotted lines are the growth rates predicted by Equation (85). The dashed lines are the numerical growth rates for modes with $k=0$.

values of $R_{m} \lesssim 0.1 R_{j}$, the results are nearly identical to those we described above for the fastest growing mode $\left(R_{\text {sing }}=\right.$ $1.1 R_{j}$, solid lines). This is to be expected based on the results shown in Figure 6 for Model C, which has $R_{m}=0.18 R_{j}$. With increasing $R_{m}$, however, the $k=0$ modes become less unstable than the modes with $R_{\text {sing }} \sim R_{j}$. By $R_{m} \sim 0.3 R_{j}$, the $k=0$ modes are fully stable, thus explaining the stability of Model B as $k \rightarrow 0$ (Figure 4 ).

\subsection{Spatial Growth of Unstable Modes}

The discussion so far was limited to modes with real $k$ and complex $\omega$. An equally interesting problem is to consider modes with real $\omega$ and complex $k$. From Equation (41), we see that the eigenfunctions take the form

$$
\begin{aligned}
\vec{E}_{1}= & {\left[E_{1 R}(R) \hat{R}+E_{1 \phi}(R) \hat{\phi}+E_{1 z}(R) \hat{z}\right] } \\
& \times \exp [-i \omega t+i m \phi+i \operatorname{Re}(k) z-\operatorname{Im}(k) z] \\
\propto & \exp [i \operatorname{Re}(k) z] \exp (z / Z),
\end{aligned}
$$

where $\omega$ is real and $Z=-1 / \operatorname{Im}(k)$ is the scale length on which the mode $e$-folds in the $z$-direction. Such spatially growing "convected" modes are particularly relevant for sources with long-lived steady jets.

As discussed in Payne \& Cohn (1985) and Appl \& Camenzind (1992), there is a strong symmetry between modes with real $k$ and complex $\omega$, and those with complex $k$ and real $\omega$. In particular, the growth rates of the two kinds of modes are related by

$$
\operatorname{Im}(k)=-\operatorname{Im}(\omega) / v_{g},
$$

where $v_{g}=\partial \operatorname{Re}(\omega) / \partial \operatorname{Re}(k)$ is the group velocity of the mode. Our unstable $m=1$ modes have $v_{g}$ very nearly equal to $c$. Therefore, we immediately obtain from Equation (85) the following estimate for the spatial $e$-folding scale $Z$ of the fastest growing convected mode:

$$
\text { Mode with } R_{\text {sing }}=1.1 R_{\mathrm{j}}: \quad \frac{Z}{R_{j}} \approx \frac{2.5 \gamma_{j}}{\left(1-2 R_{m} / R_{j}\right)} .
$$

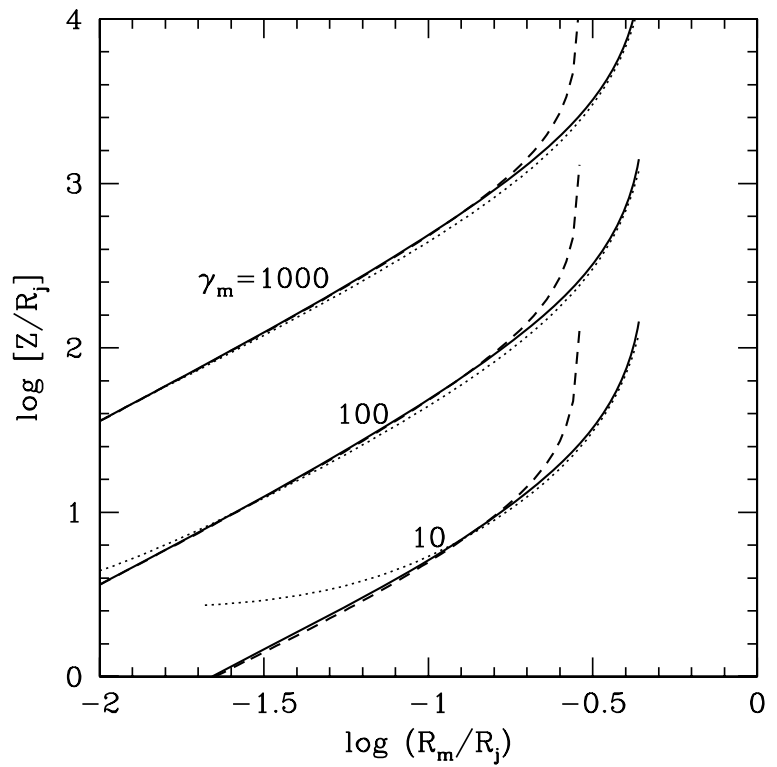

Figure 11. Numerically calculated $e$-folding scales $Z$ for modes with $m=1$, $\omega$ real and $R_{\text {sing }}=1.1 R_{j}$. These modes have among the largest growth rates. The solid lines show results for a series of models with fixed $\gamma_{m}$ and varying $R_{m} / R_{j}$. The dotted lines are the growths predicted by Equation (88). Note the very good agreement except near the bottom of the plot, where the models are nonrelativistic. The dashed lines are the numerical values of $Z$ for modes with $\omega=0$.

Zero-frequency modes $(\omega=0)$ should have almost the same $Z$ for small values of $R_{m} / R_{j}$, but the growth should cut off at a somewhat smaller value of $R_{m}$ compared to the modes with $R_{\text {sing }}=1.1 R_{j}$.

Figure 11 shows numerical results. Modes with $R_{\text {sing }}=1.1 R_{j}$ have growths consistent with Equation (88), and the modes with $\omega=0$ have similar growths except that the instability cuts off at somewhat smaller values of $R_{m}$. The results are as expected and are very similar to those shown in Figure 9.

The above results correspond to an idealized cylindrical jet. In the case of real jets we must allow for a finite opening angle $\theta_{j} \equiv d R_{j} / d z$. Many force-free jet models have opening angles that vary inversely as the Lorentz factor: $\theta_{j} \sim \mathrm{few} / \gamma_{j}$ (TMN08). Using this scaling we can estimate approximately the evolution of the mode amplitude $a$ with distance:

$$
\begin{aligned}
\frac{d a}{d R_{j}}= & \frac{1}{\theta_{j}} \frac{d a}{d z} \approx \frac{\gamma_{j}}{\text { few }} \frac{d a}{d z} \approx \frac{\gamma_{j}}{\text { few }} \frac{a}{Z} \approx \frac{\gamma_{j}}{\text { few }} \frac{a}{2.5 \gamma_{j} R_{j}} \\
& \approx \frac{1}{\text { few } \times 2.5} \frac{a}{R_{j}} .
\end{aligned}
$$

Solving this differential equation, and using $R_{j} \propto z^{\alpha / 4} \sim z^{0.5-1}$ (Equation (7)), we obtain

$$
a(z) \propto z^{\epsilon},
$$

where $\epsilon \lesssim 0.1$. This estimate is very crude, but it does suggest that, in realistic jets, the unstable kink mode we have studied in this paper grows only weakly with increasing distance.

\subsection{Toward an Improved Instability Criterion}

In Section 1, we introduced three different instability criteria, of which the IPL and TMT criteria refer specifically to rotating force-free jets. Since $B_{z}$ is practically constant in our equilibria, all of our models are close to the boundary between stability 
and instability according to the IPL criterion (Equation (2)). Similarly, since $B_{\phi}^{2} \approx \Omega^{2} R^{2} B_{p}^{2} / c^{2}$ in our equilibria, our models are marginally stable according to the TMT criterion (Equation (3)). ${ }^{13}$ It is thus not possible to understand from either of these criteria why Model A is stable and Models B and C are unstable.

It is, of course, not surprising that the above instability criteria fail. Our jet equilibria include the effects of poloidal field curvature, which were not considered by the previous authors. For easier comparison with previous work, let us rewrite our balance condition (23) as follows:

$$
\frac{1}{R^{2}} \frac{d}{d R}\left[\frac{\left(B_{0 \phi}^{2}-E_{0 R}^{2}\right) R^{2}}{8 \pi}\right]=-\frac{d}{d R}\left(\frac{B_{0 z}^{2}}{8 \pi}\right)+\left(\frac{E_{0 R}^{2}-B_{0 z}^{2}}{4 \pi R_{c}}\right) .
$$

If we leave out the last term, this is equivalent to Equation (6) in Istomin \& Pariev (1996) and Equation (16) in Lyubarskii (1999). The IPL instability criterion states that the quantity $d B_{0 z}^{2} / d R$ should be negative. We might wish to generalize this by saying that the right-hand side of Equation (91), including the poloidal curvature term, should be positive. Unfortunately, this simple modification is not sufficient since the right-hand side is positive for all of our models, whereas not all our models are unstable; not only should the right-hand side be positive, its magnitude should be larger than some amount. The same seems to be true with the TMT criterion. This criterion indicates that all our equlibria should be unstable, whereas only some of them are.

Qualitatively, what distinguishes the unstable Models B and $\mathrm{C}$ from the stable Model $\mathrm{A}$ is that the former have made the transition to the second acceleration regime, where poloidal field curvature has a strong effect on transverse force balance. This is reflected in the $\gamma(R)$ profiles (Figure 2) which have $d \gamma / d R<0$ at larger radii. Thus, one might guess that instability requires the jet to be in the second acceleration regime, i.e., poloidal field curvature to be important, or the jet to have a declining $\gamma(R)$. Once again, these related conditions by themselves are not sufficient. To have an instability, $\gamma(R)$ should decline over a sufficiently broad range of radius, e.g., $R_{m}$ should be less than $\sim 0.45 R_{j}$ in our models.

An alternate approach which we have found useful is to focus on the left-hand side of Equation (91). From Equations (17)(19), we see that for our equilibria we have $B_{0 \phi}^{2}-E_{0 R}^{2}=$ $\left(R / R_{m}\right)^{4} B_{0 z}^{2}$. Furthermore, we have seen that modes with $R_{\text {sing }} \sim R_{j}$ (the fastest growing modes) are unstable so long as $R_{m} \lesssim 0.45 R_{j}$, while modes with $k \rightarrow 0$ (long-wavelength modes) are unstable for $R_{m} \lesssim 0.3 R_{j}$. From this, we obtain the following approximate instability criteria:

$$
\begin{gathered}
\text { Modes with } R_{\text {sing }} \sim R_{\mathrm{j}}:\left(B_{0 \phi}^{2}-E_{0 R}^{2}\right)^{1 / 2}>5\left|B_{0 z}\right|, \\
\text { Modes with } k \rightarrow 0:\left(B_{0 \phi}^{2}-E_{0 R}^{2}\right)^{1 / 2}>12\left|B_{0 z}\right|,
\end{gathered}
$$

where we have set $R=R_{j}$ to obtain the numerical coefficients on the right. These conditions are easier to interpret if we boost to the comoving frame of the fluid (V. Pariev 2008, private communication), where the electric field vanishes. In this frame, the criterion for instability becomes

$$
\text { Modes with } R_{\text {sing }} \sim R_{\mathrm{j}}:\left|B_{0 \phi, \text { comov }}\right|>5\left|B_{0 z, \text { comov }}\right|,
$$

\footnotetext{
13 A strict application of the TMT criterion would indicate that our models are unstable, since $B_{\phi}^{2}>E^{2}=\Omega^{2} R^{2} B_{p}^{2} / c^{2}$. However, $B_{\phi}^{2}-E^{2} \ll B_{\phi}^{2}$, so the models deviate only slightly from marginal stability.
}

Modes with $k \rightarrow 0:\left|B_{0 \phi, \text { comov }}\right|>12\left|B_{0 z, \text { comov }}\right|$.

That is, in the comoving frame, the toroidal field must dominate the poloidal field by more than a certain critical factor. ${ }^{14}$ Written in this form, the condition resembles the KS criterion (Equation (1)).

\section{SUMMARY AND DISCUSSION}

The relativistic jet model we have considered in this paper is particularly simple: it is cylindrical, it assumes force-free conditions, and it assumes rigid rotation. Within the limitations of these reasonable approximations, we have attempted to be as close to numerically simulated jets as possible. A unique feature of our model is that we include the effect of poloidal field curvature, which is known to play an important role in numerical force-free jets (Section 2.1). Also, we choose functional forms for the various field components in the equilibrium (Section 2.2) to match as closely as possible our previous force-free simulations (TMN08).

Our equilibrium model is described by two parameters: the maximum Lorentz factor, $\gamma_{m}$, and the radius at which this maximum is achieved, $R_{m}$. The ratio of the latter to the jet radius, $R_{j}$, determines the basic physics of the equilibrium. Models in which $R_{m} / R_{j}>1$ have $\gamma(R)$ increasing monotonically with radius $R$ out to some maximum Lorentz factor $\gamma_{j}<\gamma_{m}$ at the outer edge of the jet. Model A (Figure 2) is an example. In these models the entire jet is in the first acceleration regime (see Sections 2.1, 2.2.1 and TMN08 for details). We find that all these models are perfectly stable.

Models with $R_{m} / R_{j}<1$ are more interesting. Here, $\gamma(R)$ increases up to a maximum value $\gamma_{m}$ at $R=R_{m}$ and then decreases down to a Lorentz factor $\gamma_{j}<\gamma_{m}$ at $R=R_{j}$. Models $\mathrm{B}$ and $\mathrm{C}$ (Figure 2) are examples. In these models, the jet fluid at $R<R_{m}$ is in the first acceleration regime, while the fluid at $R_{m}<R<R_{j}$ is in the second acceleration regime. We find that the subset of these models with $R_{m} / R_{j} \lesssim 0.45$ are linearly unstable. For $R_{m} / R_{j}$ just below 0.45 , all the unstable modes have short wavelengths in the $z$-direction: $\lambda=2 \pi / k_{z} \sim$ $2 \pi R_{j} / \gamma_{j}$. With decreasing $R_{m}$, a wider range of $k_{z}$ becomes unstable, and for $R_{m} / R_{j} \lesssim 0.3$, waves with $k_{z}=0$, i.e., with arbitrarily long wavelengths, become unstable. The latter modes are perhaps of most interest since they are likely to grow to the largest amplitudes. The numerical results are summarized in Figures 3-11.

The unstable modes we find are all kink modes with azimuthal wavenumber $m= \pm 1$. These are nonaxisymmetric modes in which the jet is distorted helically. A key feature is that, at each $z$, the center of mass of the jet is shifted away from $R=0$. It is well known that MHD configurations with toroidal fields are especially susceptible to the kink mode (Bateman 1978), and our models follow this trend. However, because our equilibria both rotate and move relativistically along $z$, the criterion for instability is different from the usual KS criterion (Equation (1)).

\footnotetext{
14 Since our equilibria assume a constant $\Omega$ for all field lines, the criteria (94) and (95) are technically valid only for such models. However, since the criteria have been written without any explicit reference to $\Omega$, they may be valid more generally even when $\Omega$ varies with $R$. Also, in the case of a spinning black hole, the most important example of a central "star" with nonconstant $\Omega$, the angular velocity of the horizon varies by only a factor of 2 between the pole and the equator (Blandford \& Znajek 1977; McKinney \& Narayan 2007a, $2007 b)$. Such a modest variation of $\Omega$ across the jet is unlikely to modify our results substantially, though this remains to be demonstrated.
} 
We find that the typical growth rate of the unstable kink mode in our jet models is given by Equation (85): the $e$-folding time is of order $\gamma_{j}$ times the light-crossing time $R_{j} / c$ across the jet. The extra factor of $\gamma_{j}$ is easily understood-it arises from time dilation. In the comoving frame of the jet (at $R=R_{j}$ ), we expect the growth timescale to be simply $R_{j} / c$. Lorentz transforming to the lab frame, this becomes $\gamma_{j} R_{j} / c$. For convected modes with a real frequency, the $e$-folding length scale is of order $\gamma_{j}$ times the jet radius $R_{j}$, the extra $\gamma_{j}$ arising in this case from Lorentz contraction. Since jets typically have opening angles $\sim$ few $/ \gamma_{j}$, the net result is that the unstable modes grow only slowly with distance from the base of the jet (Equation (90)). Of course, relativistic jets in astrophysical sources propagate over many decades, so in principle even this slow growth might lead to a noticeable perturbation amplitude. Nevertheless, the fact that the growth is very slow reduces the seriousness of the kink instability.

Our jet equilibria turn out to be close to the boundary between stability and instability according to either the IPL or the TMT criterion (Equations (2) and (3)), so these criteria are not useful for interpreting the results. In addition, since our models include the effects of poloidal field curvature, they lie outside the range of validity of the IPL and TMT criteria. The most useful instability criterion we have come up with is that, in the comoving frame of the jet fluid, the tangential field should be an order of magnitude or more larger than the poloidal field (Equations (94) and (95)). Expressed thus, the criterion is similar to the KS criterion (1). However, we should not apply the KS criterion in the lab frame. Rather, we should apply it in the comoving frame of the jet fluid, which is reasonable, and we should set $z \sim R_{j}$, which is again reasonable because of Lorentz contraction $\left(z \sim \gamma_{j} R_{j}\right.$ in the lab frame implies $z \sim R_{j}$ in the comoving frame).

All the work described here assumes a rigid wall enclosing the jet at the boundary $R=R_{j}$. We have done some calculations with a constant pressure boundary and we find unstable modes with much larger growth rates compared to the rigid wall case. However, since we are dealing with a force-free jet, it is not clear that a constant pressure boundary is particularly meaningful. For instance, if the pressure is from a nonrelativistic gaseous envelope or cocoon, the gas would have substantial inertia and would probably behave to first approximation like a rigid wall.

Various authors have discussed mechanisms by which instabilities might be suppressed in astrophysical jets. Hardee et al. (2007, and references therein) have shown that an external wind or cocoon can stabilize the Kelvin-Helmholtz mode in MHD jets, though it is not clear if this is relevant for force-free jets. Moll et al. (2008) show that lateral expansion causes instabilities to grow more slowly. In a sense, we have already included this effect when we derived the growth-rate estimate given in Equation (90). In addition, we note that some of the growth suppression seen by Moll et al. is probably because expansion causes different parts of the jet to lose causal contact with one other. This is not an issue for force-free models, where signals propagate at the speed of light.

It would be interesting to simulate numerically the unstable modes described in this paper. Apart from verifying the linear theory, such calculations will reveal the nonlinear development of the mode. Does the kink mode saturate at a finite amplitude and lead to a more-or-less coherent helical pattern or does it destroy the initial equilibrium? This important question can be answered only with three-dimensional simulations. Since the kink mode involves lateral motion of the jet across the axis $R=0$, the numerical technique used must be flexible enough to allow such motions (e.g., as described by McKinney \& Blandford 2009).

We conclude by reminding the reader that the work described here refers to a particularly simple model of relativistic jets which is based on the force-free approximation. In real jets, once the flow crosses the fast magnetosonic point, the inertia of the gas starts to play a role and the force-free approximation is no longer valid (e.g., Tchekhovskoy et al. 2009; Lyubarsky 2009). We must then consider the full MHD equations.

The authors thank Alison Farmer for assistance during the early stages of this work and Jonathan McKinney for numerous helpful discussions and comments on the paper. This work was supported in part by NASA grant NNX08AH32G.

\section{APPENDIX A}

\section{CONSTANCY OF POLOIDAL MAGNETIC FIELD ACROSS FORCE-FREE JETS}

Figure 1 shows that in numerical simulations of force-free jets $B_{p}$ hardly changes with $R$. Here we show that this is a common feature of all jet solutions that smoothly connect to a spinning compact object at the base.

Consider the force balance equation, Equation (8). Sufficiently near the compact object, where the jet is in the first acceleration regime (see Section 2.1), we can drop the terms proportional to $R_{c}^{-1}$ and $\left(B_{\phi}^{2}-E^{2}\right)$ since in the first acceleration regime $\gamma_{1}^{2} \ll \gamma_{2}^{2}$ leading to $E^{2} / B_{p}^{2} \ll R_{c} / R$ and $B_{\phi}^{2}-E^{2} \ll B_{p}^{2}$ (TMN08). Then the force balance equation, Equation (8), simplifies to

$$
\frac{d\left(B_{p}^{2}\right)}{d R} \approx 0 .
$$

Therefore, sufficiently near the compact object the poloidal field is nearly constant,

$$
B_{p}(R) \approx \text { const. }
$$

Each field line is labeled by the amount of poloidal magnetic flux $\Phi$ that it encloses. Due to Equation (A2) this flux can be written simply as

$$
\Phi \approx \pi B_{p} R^{2} .
$$

These relations are valid throughout the first acceleration regime. We now show that they actually hold asymptotically in all parts of the jet.

Recall that in force-free magnetospheres the enclosed poloidal current $I$ is preserved along each field line (Mestel 1961; Okamoto 1978; Thorne et al. 1986; Beskin 1997; Narayan et al. 2007; Tchekhovskoy et al. 2008),

$$
I=I(\Phi)=\frac{c}{2} R B_{\phi} \approx-\frac{\Omega}{2} B_{p} R^{2},
$$

where the approximate equality is due to $B_{\phi} \approx-E=$ $-\Omega R B_{p} / c$ for $R \gg R_{\mathrm{A}}$ (see Equations (10) and (12)). Comparing Equations (A3) and (A4) and recalling that $\Omega$ is conserved along field lines, we obtain

$$
\Phi \approx-\frac{2 \pi}{\Omega} I(\Phi) .
$$

Since both sides of this relation depend only on $\Phi$, this relation is valid everywhere in the solution, even though we derived it only in the first acceleration regime. Using Equation (A4) to 
substitute for $I$ yields back Equation (A3). Thus Equations (A2) and (A3) are valid everywhere in the jet.

\section{APPENDIX B}

\section{COEFFICIENTS IN EQUATIONS (56) AND (57)}

Here, we give explicit expressions for the coefficients $D_{1}(R)$ $D_{6}(R)$ defined in Section 3.1. The functions are

$$
\begin{gathered}
D_{1}(R)=-\frac{c k}{\omega} g-\frac{c m}{\omega R} g C_{1}, \\
D_{2}(R)=-\frac{c m}{\omega R^{2}} f-\frac{c m}{\omega R} f^{\prime}+C_{2} C_{1}-\frac{c m}{\omega R} g C_{1}^{\prime}, \\
D_{3}(R)=\left(\frac{i c k^{2}}{\omega}+\frac{i c m^{2}}{\omega R^{2}}-\frac{i \omega}{c}\right) g \\
D_{4}(R)=\frac{c k}{\omega} f+\frac{c m}{\omega R} g-h-C_{3} \frac{D_{3}}{D_{1}}, \\
D_{5}(R)=C_{4}+C_{3} \frac{D_{2}^{2}}{D_{1}^{2}}+\left(C_{6}+\frac{C_{5}}{R}\right) C_{1}+C_{5} C_{1}^{\prime}-C_{7} \frac{D_{2}}{D_{1}} \\
+C_{3}\left(\frac{D_{2} D_{1}^{\prime}}{D_{1}^{2}}-\frac{D_{2}^{\prime}}{D_{1}}\right)+\frac{i c}{\omega} g C_{1}^{\prime \prime}, \\
D_{6}(R)=C_{8}+C_{3} \frac{D_{2} D_{3}}{D_{1}^{2}}-C_{7} \frac{D_{3}}{D_{1}}+C_{3}\left(\frac{D_{3} D_{1}^{\prime}}{D_{1}^{2}}-\frac{D_{3}^{\prime}}{D_{1}}\right),
\end{gathered}
$$

where primes denote derivatives with respect to $R$, and the functions $C_{1}(R)-C_{8}(R)$ are given by

$$
\begin{gathered}
C_{1}(R)=\frac{\omega R g-c m h}{\omega R f-c k R h} . \\
C_{2}(R)=\frac{c k}{\omega R} f-\frac{c m}{\omega R^{2}} g-\frac{h}{R}-\frac{h}{R_{c}}+\frac{c k}{\omega} f^{\prime}-h^{\prime}, \\
C_{3}(R)=\frac{i c}{\omega}\left(f+g C_{1}\right), \\
C_{4}(R)=-\frac{i c m^{2}}{\omega R^{2}} f+\frac{i \omega}{c} f+\frac{i c k m}{\omega R} g-i k h, \\
C_{5}(R)=\frac{i c}{\omega R} g+\frac{i c}{\omega R_{c}} g+\frac{i c}{\omega} g^{\prime},
\end{gathered}
$$

$$
\begin{gathered}
C_{6}(R)=\frac{i c k m}{\omega R} f-\frac{i c k^{2}}{\omega} g-\frac{2 i c}{\omega R^{2}} g \\
+\frac{i \omega}{c} g-\frac{i m}{R} h \\
C_{7}(R)=\frac{2 i c}{\omega R} f+\frac{i c}{\omega} f^{\prime}+C_{5} C_{1}+\frac{2 i c}{\omega} g C_{1}^{\prime}, \\
C_{8}(R)=\frac{2 c k}{\omega R} f-\frac{c m}{\omega R^{2}} g-\frac{2 h}{R}+\frac{c m}{\omega R_{c} R} g-\frac{h}{R_{c}}+\frac{c k}{\omega} f^{\prime} \\
+\frac{c m}{\omega R} g^{\prime}-h^{\prime} .
\end{gathered}
$$

\section{REFERENCES}

Appl, S., \& Camenzind, M. 1992, A\&A, 256, 354

Appl, S., Lery, T., \& Baty, H. 2000, A\&A, 355, 818

Bateman, G. 1978, MHD Instabilities (Cambridge, MA: MIT Press)

Begelman, M. C. 1998, ApJ, 493, 291

Benford, G. 1981, ApJ, 247, 792

Beskin, V. S. 1997, Sov. Phys. Usp., 40, 659

Blandford, R. D., \& Znajek, R. L. 1977, MNRAS, 179, 433

Ferrari, A., Trussoni, E., \& Zaninetti, L. 1978, A\&A, 64, 43

Gammie, C. F., McKinney, J. C., \& Tóth, G. 2003, ApJ, 589, 444

Goldreich, P., Goodman, J., \& Narayan, R. 1986, MNRAS, 221, 339

Goldreich, P., \& Julian, W. H. 1969, ApJ, 157, 869

Goldreich, P., \& Tremaine, S. 1978, ApJ, 222, 850

Hardee, P., Mizuno, Y., \& Nishikawa, K. I. 2007, Ap\&SS, 311, 281

Istomin, Y. N., \& Pariev, V. I. 1996, MNRAS, 281, 1

Kadomtsev, B. B. 1966, Rev. Plasma Phys., 2, 153

Lery, T., Baty, H., \& Appl, S. 2000, A\&A, 355, 1201

Li, L. X. 2000, ApJ, 531, L111

Lynden Bell, D. 2006, MNRAS, 369, 1167

Lyubarskii, Y. E. 1999, MNRAS, 308, 1006

Lyubarsky, Y. 2009, ApJ, submitted (arXiv:0902.3357)

McKinney, J. C. 2006, MNRAS, 368, 1561

McKinney, J. C., \& Blandford, R. D. 2009, MNRAS, 394, L126

McKinney, J. C., \& Gammie, C. F. 2004, ApJ, 611, 977

McKinney, J. C., \& Narayan, R. 2007a, MNRAS, 375, 513

McKinney, J. C., \& Narayan, R. 2007b, MNRAS, 375, 531

Mestel, L. 1961, MNRAS, 122, 473

Mignone, A., \& McKinney, J. C. 2007, MNRAS, 378, 1118

Mizuno, Y., Hardee, P., \& Nishikawa, K. I. 2007, ApJ, 662, 835

Moll, R., Spruit, H. C., \& Obergaulinger, M. 2008, A\&A, 492, 621

Narayan, R., Goldreich, P., \& Goodman, J. 1987, MNRAS, 228, 1

Narayan, R., McKinney, J. C., \& Farmer, A. J. 2007, MNRAS, 375, 548

Okamoto, I. 1978, MNRAS, 185, 69

Payne, D. G., \& Cohn, H. 1985, ApJ, 291, 655

Ruderman, M. A., \& Sutherland, P. G. 1975, ApJ, 196, 51

Tchekhovskoy, A., McKinney, J. C., \& Narayan, R. 2007, MNRAS, 379, 469

Tchekhovskoy, A., McKinney, J. C., \& Narayan, R. 2008, MNRAS, 388, 551

Tchekhovskoy, A., Mc Kinney, J. C., \& Narayan, R. 2009, ApJ, submitted (arXiv:0901.4776)

Thorne, K. S., Price, R. H., \& Mac Donald, D. A. 1986, Black Holes: The Membrane Paradigm (New Haven, CT: Yale Univ. Press)

Tomimatsu, A., Matsuoka, T., \& Takahashi, M. 2001, Phys. Rev. D, 64, 123003 